\title{
Activation of mitochondrial unfolded protein response protects against multiple exogenous stressors
}

\author{
Sonja K Soo ${ }^{1,2}$, Annika Traa ${ }^{1,2}$ (D), Paige D Rudich ${ }^{1,2}$ (D), Meeta Mistry ${ }^{3}$, Jeremy M Van Raamsdonk ${ }^{1,2,4,5}$ (B)
}

\begin{abstract}
The mitochondrial unfolded protein response (mitoUPR) is an evolutionarily conserved pathway that responds to mitochondria insults through transcriptional changes, mediated by the transcription factor ATFS-1/ATF-5, which acts to restore mitochondrial homeostasis. In this work, we characterized the role of ATFS-1 in responding to organismal stress. We found that activation of ATFS-1 is sufficient to cause up-regulation of genes involved in multiple stress response pathways including the DAF-16mediated stress response pathway, the cytosolic unfolded protein response, the endoplasmic reticulum unfolded protein response, the SKN-1-mediated oxidative stress response pathway, the HIF-1-mediated hypoxia response pathway, the p38mediated innate immune response pathway, and antioxidant genes. Constitutive activation of ATFS- 1 increases resistance to multiple acute exogenous stressors, whereas disruption of atfs-1 decreases stress resistance. Although ATFS-1-dependent genes are up-regulated in multiple long-lived mutants, constitutive activation of ATFS-1 decreases lifespan in wild-type animals. Overall, our work demonstrates that ATFS-1 serves a vital role in organismal survival of acute stressors through its ability to activate multiple stress response pathways but that chronic ATFS-1 activation is detrimental for longevity.
\end{abstract}

DOI 10.26508/lsa.202101182 | Received 30 July 2021 | Revised 10 September 2021 | Accepted 20 September 2021 | Published online 28 September 2021

\section{Introduction}

The mitochondrial unfolded protein response (mitoUPR) is a stress response pathway that acts to reestablish mitochondrial homeostasis by inducing transcriptional changes in genes involved in the metabolism and restoration of mitochondrial protein folding (Zhao et al, 2002). Various perturbations to the mitochondria can activate mitoUPR, including disruption of mitochondrial translation, disruption of mitochondrial protein synthesis, impairment of oxidative phosphorylation, disruption of mitochondrial proteostasis, altered metabolism, defects in mitochondrial DNA, excess reactive oxygen species (ROS), disruption of protein degradation, and defects in mitochondrial import (Shpilka \& Haynes, 2018). The mitoUPR is mediated by the transcription factor activating transcription factor associated with stress-1 (ATFS-1) in Caenorhabditis elegans (Nargund et al, 2012), and activating transcription factor 5 (ATF5) in mammals (Fiorese et al, 2016).

ATFS-1/ATF5 regulates the mitoUPR through its dual targeting domains: a mitochondrial targeting sequence (MTS) and a NLS. Under normal unstressed conditions, the MTS causes ATFS-1 to enter the mitochondria through the HAF-1 import channel. Inside the mitochondria, ATFS-1 is degraded by the protease CLPP-1/CLP1 (Nargund et al, 2012). However, when mitochondrial import or degradation of ATFS-1 is disrupted under conditions of mitochondrial stress, ATFS-1 accumulates in the cytoplasm. The NLS of the cytoplasmic ATFS-1 then targets it to the nucleus, where ATFS-1 acts with the transcription factor DVE-1 and transcriptional regulator UBL-5 to up-regulate expression of chaperones, proteases, and other proteins (Jovaisaite et al, 2014).

In order to study the role of the mitoUPR in longevity, we previously disrupted atfs- 1 in long-lived nuo- 6 mutants, which contain a point mutation that affects complex I of the electron transport chain (Yang \& Hekimi, 2010b). nuo-6 mutants have a mild impairment of mitochondrial function that leads to increased lifespan and enhanced resistance to multiple stressors. We found that loss of atfs- 1 not only decreased the lifespan of nuo- 6 worms but also abolished the increased stress resistance of these worms, thereby suggesting that ATFS-1 contributes to both longevity and stress resistance in these worms (Wu et al, 2018).

Although a role for the mitoUPR in longevity has been reported (Durieux et al, 2011; Houtkooper et al, 2013; Berendzen et al, 2016; Merkwirth et al, 2016) and debated (Bennett et al, 2014; Bennett \& Kaeberlein, 2014), little is known about the role of ATFS-1 in response to exogenous stressors. Activation of ATFS-1 can increase organismal resistance to the pathogenic bacterium Pseudomonas aeruginosa (Pellegrino et al, 2014) and can protect against anoxiareperfusion-induced death (Pena et al, 2016).

In this study, we use $C$. elegans to define the relationship between ATFS-1 and organismal stress resistance and to explore the

${ }^{1}$ Department of Neurology and Neurosurgery, McGill University, Montreal, Canada ${ }^{2}$ Metabolic Disorders and Complications Program, and Brain Repair and Integrative Neuroscience Program, Research Institute of the McGill University Health Centre, Montreal, Canada ${ }^{3}$ Bioinformatics Core, Harvard School of Public Health, Harvard Medical School, Boston, MA, USA ${ }^{4}$ Division of Experimental Medicine, Department of Medicine, McGill University, Montreal, Canada ${ }^{5}$ Department of Genetics, Harvard Medical School, Boston, MA, USA 
underlying mechanisms. We find that activation of ATFS-1 is sufficient to up-regulate genes from multiple stress response pathways and is important for transcriptional changes induced by oxidative stress and bacterial pathogen exposure. Constitutive activation of ATFS-1 is also sufficient to increase resistance to multiple external stressors. Although ATFS-1-dependent genes are up-regulated in several long-lived mutants that are representative of multiple pathways of lifespan extension, chronic activation of ATFS-1 does not extend longevity. Overall, our results demonstrate a crucial role for ATFS-1 in organismal stress response through activation of multiple stress response pathways.

\section{Results}

\section{ATFS-1 activates genes from multiple stress response pathways}

Mild impairment of mitochondrial function by a mutation in nuo- 6 results in the activation of the mitoUPR. We previously performed a bioinformatics analysis of genes that are up-regulated in nuo- 6 mutants in an ATFS-1-dependent manner and discovered an enrichment of genes associated with the GO term "response to stress" (Wu et al, 2018). Based on this observation, we hypothesized that ATFS-1 may be able to activate other stress response pathways. To test this hypothesis, we quantified the expression of established target genes from eight different stress response pathways under conditions where ATFS-1 is either activated or disrupted.

For this analysis, we picked target genes that have been commonly used in the literature to represent their associated stress response pathway. These target genes included hsp- 6 in the mitochondrial unfolded protein response (mitoUPR) pathway (Yoneda et al, 2004; Dues et al, 2016); hsp-4 in the ER unfolded protein response (ER-UPR) pathway (Urano et al, 2002; Dues et al, 2016); hsp-16.2 in the cytoplasmic unfolded protein response pathway (cytoUPR) (Link et al, 1999; Dues et al, 2016); sod-3 in the DAF-16-mediated stress response pathway (Honda \& Honda, 1999; Dues et al, 2016); gst-4 in the SKN1-mediated stress response pathway (Kahn et al, 2008; Dues et al, 2016); nhr-57 in the HIF-1-mediated hypoxia response pathway (Bishop et al, 2004; Dues et al, 2016); Y9C9A.8 in the p38-mediated innate immunity pathway (Fletcher et al, 2019; Campos et al, 2021); and trx-2, an antioxidant gene (Cacho-Valadez et al, 2012) (Table S1).

To activate ATFS-1, we used the nuo- 6 mutation. We also examined gene expression in two different gain-of-function (GOF) mutants with constitutively active ATFS-1: atfs-1(et15) and atfs1(et17). Both of these constitutively active ATFS-1 mutants have mutations in the MTS which increase nuclear localization of ATFS-1 (Rauthan et al, 2013). To identify ATFS-1-dependent genes, we used a loss-of-function (LOF) atfs-1 deletion mutation ( $\mathrm{kk3094}$ ) to disrupt ATFS-1 function in wild-type worms and nuo-6 mutants.

We found that compared with wild-type worms, atfs-1(gk3094) deletion mutants did not have decreased expression levels for the target genes of any of the stress response pathways (Fig 1). This indicates that ATFS-1 is not required for the basal expression levels of these stress response genes.

Activation of the mitoUPR through mutation of nuo- 6 resulted in significant up-regulation of target genes from the mitoUPR ( $h s p-6$;
Fig 1A), the DAF-16-mediated stress response (sod-3; Fig 1D), the SKN-1-mediated oxidative stress response (gst-4; Fig 1E), the HIF1-mediated hypoxia response ( $n$ hr-57; Fig 1F), the p38-mediated innate immunity pathway (Y9C9A.8; Fig 1G), and antioxidant defense (trx-2; Fig 1H). Importantly, for all of these genes, inhibiting the mitoUPR through disruption of atfs-1 prevented the up-regulation of the stress response in nuo-6;atfs-1(gk3094) worms (Fig 1A and $\mathrm{D}-\mathrm{H}$ ), indicating that ATFS-1 is required for the activation of these stress pathway genes during mitochondrial stress.

Constitutive activation of ATFS-1 in atfs-1(et15) mutants resulted in up-regulation of the majority of the target genes up-regulated in nuo-6 mutants, except for the SKN-1 target gene gst-4 (Fig $1 \mathrm{~A}$ and D-H). Similarly, constitutively active atfs-1(et17) mutants result in significant up-regulation of hsp-6, sod-3, Y9C9A.8, and trx-2 and a nonsignificant $77 \%$ increase in $n h r-57$ expression (Fig 1). This indicates that ATFS-1 activation is sufficient to induce up-regulation of specific stress response genes independent of mitochondrial stress. Activating the mitoUPR through the nuo- 6 mutation or through the constitutively active ATFS-1 mutants did not significantly increase the expression of the ER-UPR target gene hsp-4 (Fig 1B) or the cyto-UPR target gene hsp-16.2 (Fig 1C). However, both the nuo- 6 mutant and the constitutively active ATFS- 1 mutants had a 2.5- to 19.5-fold increase in hsp-16.2 levels, which failed to reach significance due to variability between replicates, and the fact that hsp-16.2 expression levels can be increased up to 60-fold.

As only one gene was examined per stress response pathway, it is possible that different target genes may yield a different result. In addition, some of the stress response genes that we examined are not exclusively activated by the pathway that they are frequently used to represent. For example, gst-4 is an antioxidant gene that is commonly used as a readout of SKN-1 activity but can also be activated by DAF-16 and the mitoUPR (see Table S3 for lists of genes that are up-regulated by activation of different stress response pathways).

To circumvent these potential limitations and to gain a more comprehensive view of the extent to which mitoUPR activation causes up-regulation of genes in other stress response pathways, we compared genes up-regulated in the constitutively active atfs-1 mutant, atfs-1(et15), with genes up-regulated by activation of different stress response pathways. As a proof of principle, we first examined the overlap between up-regulated genes in atfs-1(et15) mutants and genes up-regulated by activation of the mitoUPR with spg-7 RNAi in an ATFS-1-dependent manner (Nargund et al, 2012).

We identified genes up-regulated by the activation of other stress response pathways from published gene expression studies. The genes and relevant pathways are listed in Table S3. ER-UPR pathway target genes were defined as genes up-regulated by tunicamycin exposure and dependent on ire-1,xbp-1, pek-1, or atf-6 (Shen et al, 2005b). Cyto-UPR pathway genes are genes upregulated by overexpression of heat shock factor 1 (HSF-1) and genes bound by HSF-1 after a 30-min heat shock at $34^{\circ} \mathrm{C}$ ( $\mathrm{Li}$ et al, 2016; Sural et al, 2019). DAF-16 pathway genes were identified by Tepper et al by performing a meta-analysis of 46 previous gene expression studies, comparing conditions in which DAF-16 is activated (e.g., daf-2 mutants) and conditions in which the activation is inhibited by disruption of daf-16 (e.g., daf-2;daf-16 mutants) (Tepper et al, 2013). SKN-1 pathway genes were identified as genes that exhibit decreased expression in wild-type worms treated with 


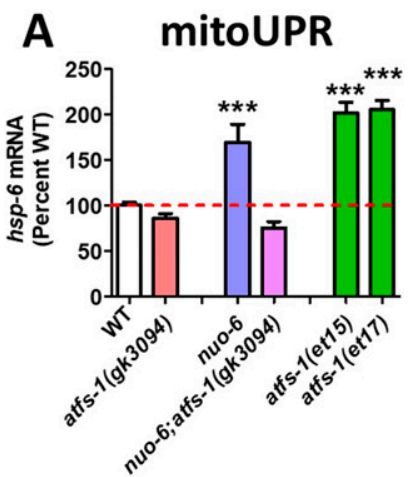

E

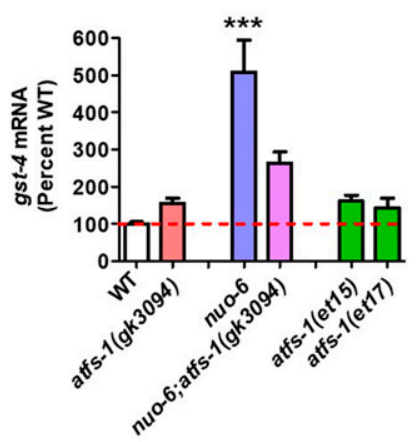

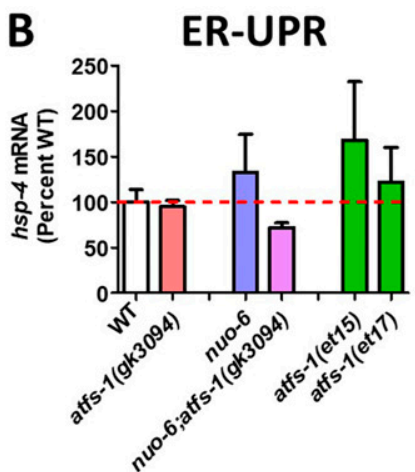

$\mathbf{F}$

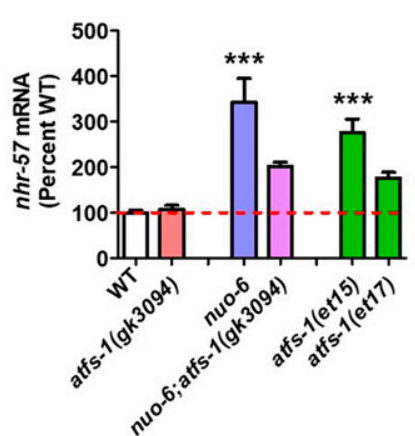

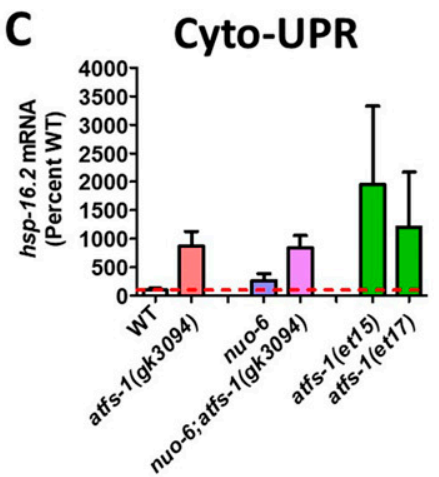
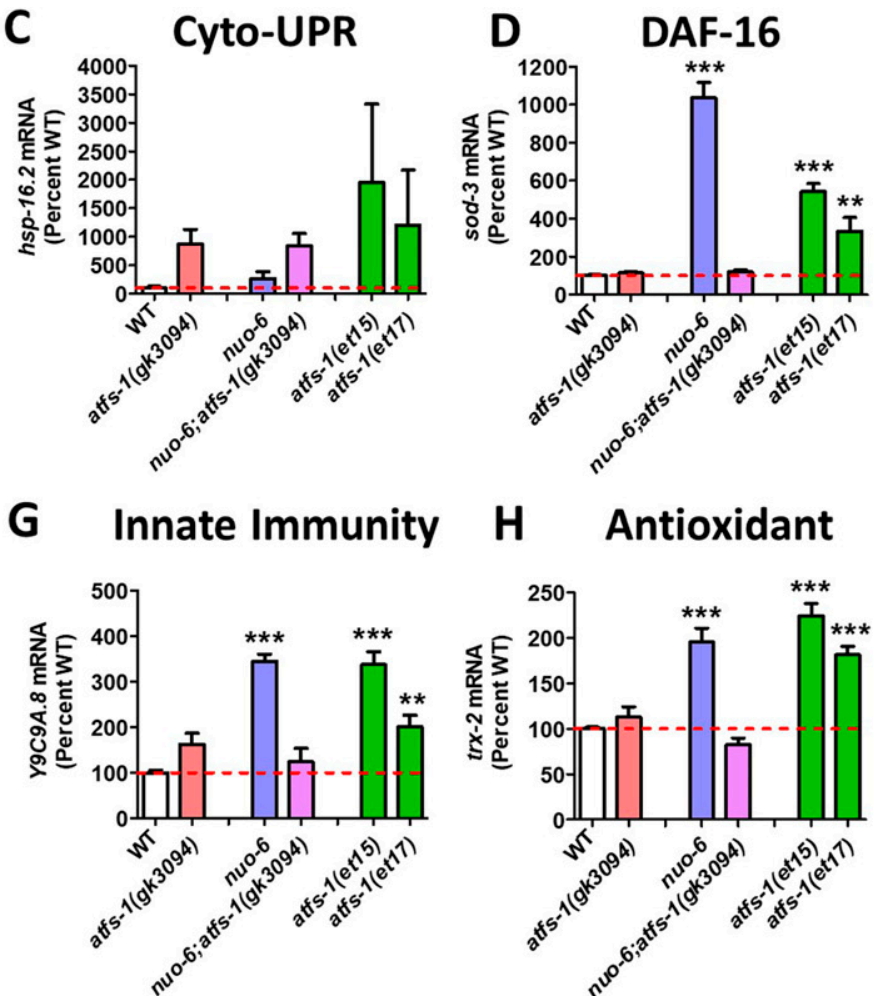

\section{H Antioxidant}

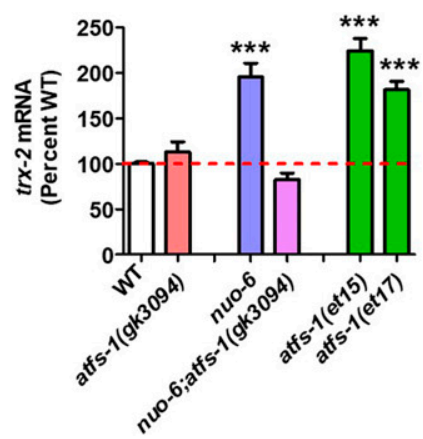

Figure 1. Activation of ATFS-1 up-regulates genes from multiple stress response pathways.

To determine the role of ATFS-1 in the activation of genes from different stress response pathways, we activated ATFS-1 by mildly impairing mitochondrial function through a mutation in nuo-6 (blue bars) and then examined the effect of disrupting atfs-1 using an atfs-1 deletion mutant atfs-1(gk3094) (purple bars). We also examined the expression of these genes in two constitutively active atfs-1 mutants, atfs-1(et15) and atfs-1(et17) (green bars). (A, B, C, D, E, F, G, H) Target genes from the mitochondrial unfolded protein response (A, mitoUPR, $h s p-6)$, the ER unfolded protein response (B, ER-UPR, hsp-4), the cytoplasmic unfolded protein response (C, Cyto-UPR, hsp-16.2), the DAF-16-mediated stress response (D, sod-3), SKN-1-mediated oxidative stress response (E, gst-4), HIF-1-mediated hypoxia response (F, nhr-57), p38-mediated innate immune pathway (G, Y9C9A.8), and antioxidant defense ( $\mathrm{H}$, trx-2) were measured. Target genes from the mitoUPR, DAF-16-mediated stress response, SKN-1-mediated oxidative stress response, HIF-1-mediated hypoxia response, p38-mediated innate immune pathway, and antioxidant defense are all significantly up-regulated in nuo6 mutants in an ATFS-1-dependent manner. Target genes from the mitoUPR, DAF-16-mediated stress response, HIF-1-mediated hypoxia response, p38-mediated innate immune pathway, and antioxidant defense are also up-regulated in at least one of the constitutively activated atfs-1 mutants. In contrast, activation of ATFS- 1 by nuo- 6 mutation or atfs-1 gain-of-function mutations did not significantly affect target gene expression for the ER-UPR or the Cyto-UPR. atfs-1(gk3094) is a loss-of-function deletion mutant. atfs-1(et15) and atfs-1(et17) are constitutively active gain-of-function mutants. A full list of genes that are up-regulated by ATFS-1 activation can be found in Table S2. Data information: Error bars indicate SEM. ${ }^{* *} P<0.01,{ }^{* *} P<0.001$. Statistical analysis was performed using a one-way ANOVA with the Bonferroni post hoc test. The number of replicates and statistical analysis can be found in Table S6.

skn-1 RNAi, genes that are up-regulated in glp-1 mutants in an SKN1-dependent manner, genes that are up-regulated by germ line stem cell removal in an SKN-1-dependent manner (Steinbaugh et al, 2015), and genes up-regulated in daf-2 mutants in an SKN-1-dependent manner (Ewald et al, 2015). HIF-1-mediated hypoxia genes are genes induced by hypoxia in an HIF-1-dependent manner (Shen et al, 2005a). Innate immunity genes are defined as genes up-regulated by exposure to P. aeruginosa strain PA14 in a PMK-1- and ATF-7dependent manner (Fletcher et al, 2019), where PMK-1 and ATF-7 are part of the p38-mediated innate immune signaling pathway. Finally, antioxidant genes include a comprehensive list of genes involved in antioxidant defense such as superoxide dismutases (sod), catalases $(c t l)$, peroxiredoxins ( $p r d x)$, or thioredoxins (trx).

In comparing genes up-regulated in the constitutively active atfs-1 mutant et15 with the previously published gene lists, we found that $51 \%$ of genes up-regulated by spg-7 RNAi in an ATFS1-dependent manner are also up-regulated by constitutive activation of ATFS-1 (Fig 2A). Similarly, there was a highly significant overlap of up-regulated genes between atfs-1(et15) mutants and each of the other stress response pathways. atfs-1(et15) had a $25 \%$ overlap with genes of the ER-UPR pathway (Fig 2B); $22 \%$ overlap with genes of the Cyto-UPR pathway (Fig 2C); $26 \%$ overlap with genes of the DAF-16-mediated stress response pathway (Fig 2D); 30\% overlap with genes of the SKN-1-mediated oxidative stress response pathway (Fig 2E); 23\% overlap with genes of the HIF-1-mediated hypoxia response pathway (Fig 2F); 22\% overlap with genes of the p38-mediated innate immunity pathway (Fig 2G); and 33\% overlap with antioxidant genes (Fig 2H). Combined, this indicates that activation of ATFS-1 is sufficient to up-regulate target genes in multiple stress response pathways.

To determine the extent to which genes common to multiple stress response pathways are up-regulated by ATFS-1 activation, we generated an UpSetR plot to simultaneously compare the overlaps between all of these gene sets. We found that there are many genes that can be up-regulated by activation of different stress response pathways (Figs $2 \mathrm{I}$ and S1A and B and Table S4). In addition, there are 


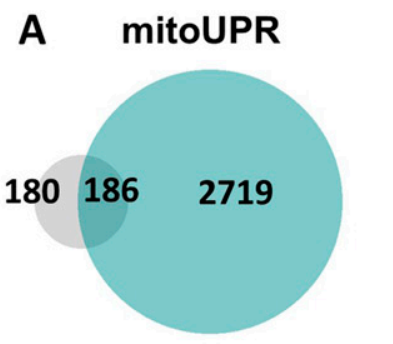

$51 \%$ overlap; $p=4.6 \times 10^{-53}$
B

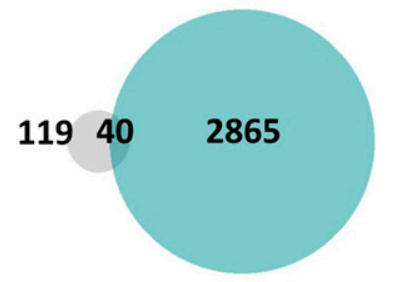

$25 \%$ overlap; $p=0.003$

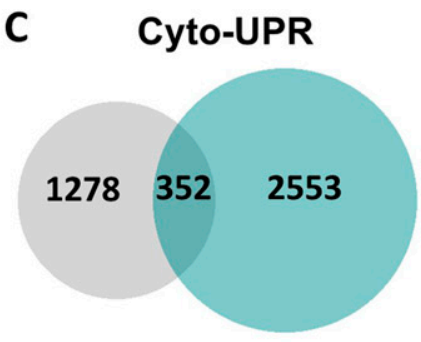

$22 \%$ overlap; $p=1 \times 10^{-8}$

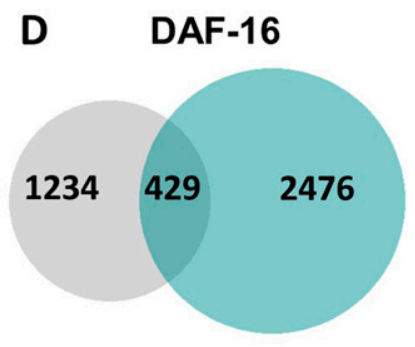

$26 \%$ overlap; $p=9 \times 10^{-25}$

\section{E SKN-1}

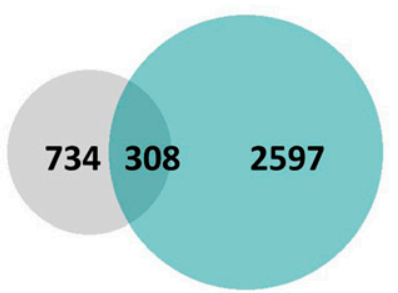

$30 \%$ overlap; $p=7.5 \times 10^{-28}$
F

HIF-1 hypoxia

4313

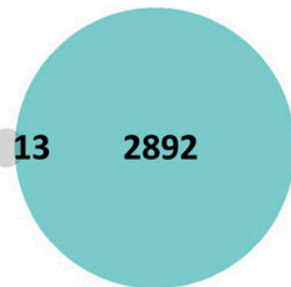

$23 \%$ overlap; $p<0.05$
G Innate Immunity

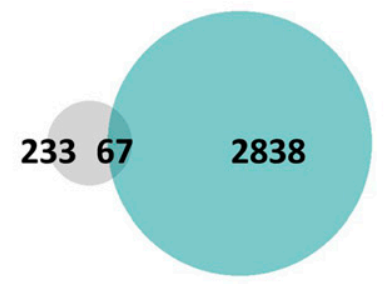

$22 \%$ overlap; $\mathbf{p}=\mathbf{0 . 0 0 5}$
H Antioxidant

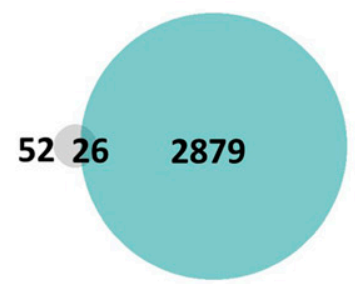

$33 \%$ overlap; $p=2.6 \times 10^{-5}$
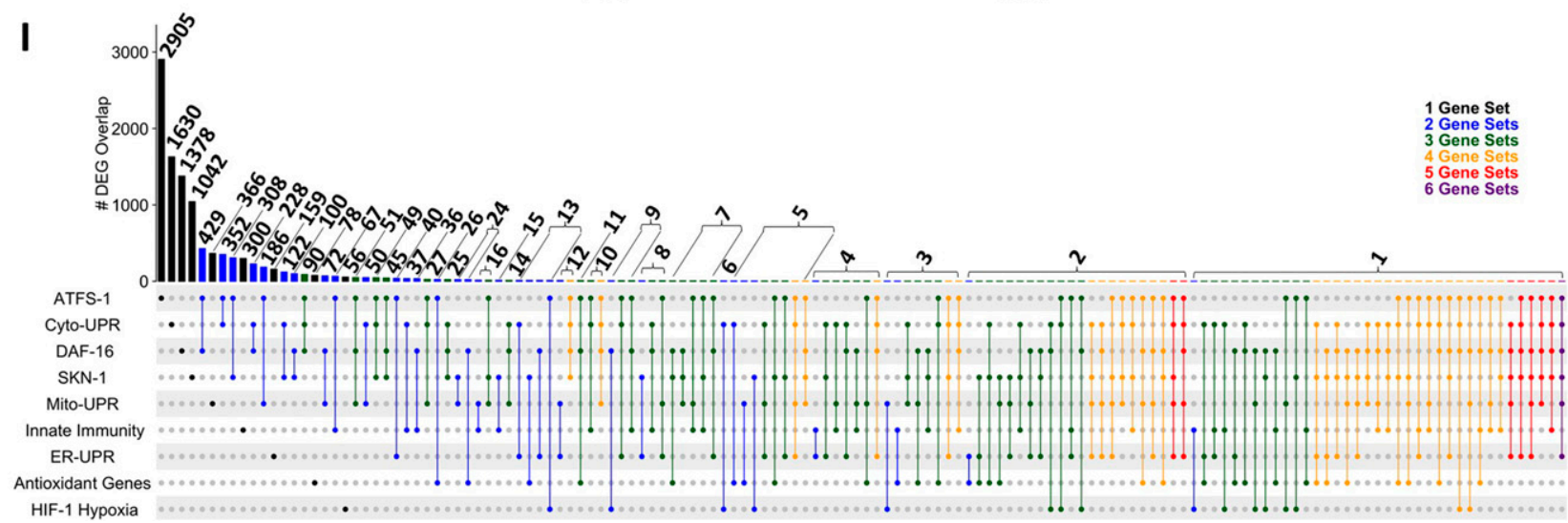

Figure 2. Constitutive activation of ATFS-1 results in up-regulation of genes from multiple stress response pathways.

(A, B, C, D, E, F, G, H) Genes that are up-regulated by constitutive activation of ATFS-1 were compared with previously published lists of genes involved in different stress response pathways, including the mitochondrial unfolded protein response (A, mitoUPR), the ER unfolded protein response (B, ER-UPR), the cytoplasmic unfolded protein response (C, Cyto-UPR), the DAF-16-mediated stress response (D), the SKN-1-mediated oxidative stress response (E), the HIF-1-mediated hypoxia response (F), the p38mediated innate immune response (G), and antioxidant genes $(\mathrm{H})$. In every case, there was a significant degree of overlap ranging from 22 to $51 \%$. Grey circles indicate genes that are up-regulated by activation of the stress response pathway indicated. Turquoise circles indicate genes that are up-regulated in the atfs-1(et15) constitutively active gain-of-function mutant. The numbers inside the circles show how many genes are up-regulated. The percentage overlap is the number of overlapping genes as a percentage of the number of genes up-regulated by the stress response pathway. P-values indicate the significance of the difference between the observed number of overlapping genes between the two gene sets, and the expected number of overlapping genes if the genes were picked at random. Panel (I) shows an inclusive UpSetR plot displaying the overlap between up-regulated genes associated with each stress response pathway. Vertical bars indicate the number of genes in common (overlap) between gene sets indicated by the dots below. Horizontal black bars indicate the number of genes within each gene set. mitouPR, mitochondrial unfolded protein response; ER-UPR, endoplasmic reticulum unfolded protein response; Cyto-UPR, cytoplasmic unfolded protein response; DAF-16, DAF16-mediated stress response pathway; SKN-1, SKN-1-mediated oxidative stress response pathway; HIF-1, HIF-1-mediated hypoxia response pathway; innate immunity, p38-mediated innate immunity pathway; antioxidant, antioxidant genes. Stress pathway gene lists and sources can be found in Table S3. Lists of genes common to multiple stress response pathways can be found in Table $\$ 4$.

multiple genes that are up-regulated by ATFS-1 activation and independent of other stress response pathways (Table S4).

\section{ATFS-1 can bind to the same promoter as other stress-responsive transcription factors}

The fact that ATFS-1 activation results in the up-regulation of the same genes as activation of other stress response pathways does not imply direct regulation of these genes by either transcription factor. ATFS-1 could modulate these genes either directly by binding to promoter or enhancer elements, or indirectly by acting on other transcription factors or altering metabolism or physiology. To gain insights into the mechanism of regulation, we sought to determine if ATFS-1 can bind to the same genes as other stressresponsive transcription factors. We compared previously published chromatin immunoprecipitation sequencing (ChIP-seq) 
experiments involving ATFS-1 (Nargund et al, 2015), HSF-1 (Kovacs et al, 2019), DAF-16 (Kumar et al, 2015; Webb et al, 2016), SKN-1 (Niu et al, 2011), HIF-1 (Kudron et al, 2018), and ATF-7 (Fletcher et al, 2019).

We found that ATFS-1 can bind to several of the same genes as other stress-responsive transcription factors (Fig S2). The degree of overlap ranged from $16 \%$ for HSF-1 to $61 \%$ for HIF-1. This suggests that ATFS-1 can directly regulate these genes. However, indirect regulation of gene expression could also contribute to the overlap in gene expression observed in Fig 2. It is important to note that these ChIP-seq experiments were performed under different conditions (e.g., ATFS-1 was examined in response to spg-7 RNAi and ATF-7 was examined in response to bacterial pathogen exposure). If these experiments were performed under the same conditions, the degree of overlap could be different from that under these specific conditions.

\section{ATFS- 1 is required for transcriptional responses to exogenous stressors}

Having shown that constitutive activation of ATFS-1 can induce upregulation of genes involved in various stress response pathways, we next sought to determine the role of ATFS-1 in the genetic response to different stressors. To do this, we exposed wild-type animals and atfs-1(gk3094) LOF mutants to six different external stressors and quantified the resulting up-regulation of stress response genes using quantitative RT-PCR (qPCR). The examined stress response genes were the established target genes of the stress response pathways that we examined in Fig 1 and genes that we previously identified as up-regulated by specific stressors using fluorescent reporter strains (Dues et al, 2016). These genes included hsp-6, hsp-4, hsp-16.2, sod-3, gst-4, nhr-57, Y9C9A.8, trx-2, ckb-2, gcs1, sod-5, T24B8.5/sysm-1, clec-67, and dod-22. We found that exposure to either oxidative stress ( $4 \mathrm{mM}$ paraquat, $48 \mathrm{~h}$ ) or the bacterial pathogen $P$. aeruginosa strain PA14 induced a significant up-regulation of stress response genes in wild-type worms, which was suppressed by disruption of atfs-1 (Figs $3 \mathrm{~A}$ and B, S3, and S4). In contrast, exposure to heat stress $\left(35^{\circ} \mathrm{C}, 2 \mathrm{~h}\right.$; Figs $3 \mathrm{C}$ and S5), osmotic stress (300 mM NaCl, 24 h; Figs 3D and S6), anoxic stress (24 h; Figs $3 \mathrm{E}$ and S7), or ER stress (tunicamycin for $24 \mathrm{~h}$; Figs $3 \mathrm{~F}$ and S8) caused up-regulation of stress response genes in both wild-type and atfs1(gk3094) worms to a similar extent, or to a greater extent in atfs-1 deletion mutants. Combined, these results indicate that ATFS-1 is required for up-regulating stress response genes in response to exposure to oxidative stress or bacterial pathogens. Although we did not observe evidence for a role of ATFS-1 in up-regulating stress response genes following exposure to other stressors, it is possible that there are genes that we did not examine that are up-regulated by the other four stressors in an ATFS-1-dependent manner.

\section{Modulation of ATFS-1 levels affects resistance to multiple stressors}

Due to the crucial role of ATFS-1 in up-regulating genes in multiple stress response pathways, we next sought to determine the extent to which activating ATFS-1 protects against exogenous stressors. We quantified resistance to stress in two constitutively active atfs-1 GOF mutants (atfs-1(et15), atfs-1(et17)) compared with wild-type worms. For comparison, we also included an atfs-1 LOF deletion mutant (atfs-1(gk3094)), which we previously found to have decreased resistance to oxidative stress, heat stress, osmotic stress, and anoxic stress (Wu et al, 2018).

Resistance to acute oxidative stress was measured by exposing worms to $300 \mu \mathrm{M}$ juglone. We found that both GOF mutants, atfs1(et15) and atfs-1(et17), have increased resistance to acute oxidative stress compared with wild-type worms, while atfs-1(gk3094) deletion mutants were less resistant compared to wild-type worms (Fig 4A). To quantify resistance to chronic oxidative stress, worms were transferred to plates containing $4 \mathrm{mM}$ paraquat beginning at day 1 of adulthood until death. Similar to the acute assay, atfs1(et17) mutants were more resistant to chronic oxidative stress, whereas atfs-1(gk3094) mutants were less resistant to chronic oxidative stress compared to wild-type worms (Fig 4B). Oddly, atfs1(et15) GOF mutants exhibited decreased resistance to chronic oxidative stress. The diminished protection in atfs-1(et17) mutants and lack of protection in the atfs-1(et15) mutants in the paraquat assay may be due to the chronic nature of the assay, compared with the juglone assay which measures resistance to acute oxidative stress.

Resistance to heat stress was measured by incubating worms at $37^{\circ} \mathrm{C}$. None of the mutants showed increased survival during heat stress, with both atfs-1(et15) and atfs-1(gk3094) mutants exhibiting a significant decrease in survival compared with wild-type worms (Fig 4C). Resistance to ER stress was measured by exposing worms to $50 \mu \mathrm{g} / \mathrm{ml}$ tunicamycin. We found that atfs-1(et15) and atfs-1(et17) constitutively active mutants have increased resistance to $E R$ stress, whereas atfs-1(gk3094) deletion mutants have an equivalent survival to wild-type worms (Fig 4D). Resistance to osmotic stress was quantified on plates containing $500 \mathrm{mM} \mathrm{NaCl}$ after $48 \mathrm{~h}$. Under these conditions, the constitutively active atfs-1 mutants had increased survival compared with wild-type worms, whereas atfs1(gk3094) deletion mutants had decreased survival (Fig 4E). Resistance to anoxic stress was measured by placing worms in an oxygen-free environment for $75 \mathrm{~h}$, followed by a $24-\mathrm{h}$ recovery period. We observed increased survival in atfs-1(et15) and atfs-1(et17) mutants and a trend towards decreased survival in atfs-1(gk3094) mutant compared with wild-type worms (Fig 4F).

Lastly, to test resistance to bacterial pathogens, worms were exposed to $P$. aeruginosa strain PA14 in either a fast kill assay, in which worms die from a toxin produced by the bacteria, or a slow kill assay, in which worms die due to the intestinal colonization of the pathogenic bacteria (Kirienko et al, 2014). In the fast kill assay, constitutive activation of ATFS-1 increased survival in atfs-1(et15) and atfs-1(et17) mutants compared with wild-type worms (Fig 4G). atfs-1(gk3094) deletion mutants also exhibited increased survival. For the slow kill assay, we used two established protocols: one in which the assay is initiated at the L 4 larval stage and performed at $25^{\circ} \mathrm{C}$ (Kirienko et al, 2014; Pellegrino et al, 2014; Dues et al, 2016) and the other in which the assay is initiated at day 3 of adulthood and performed at $20^{\circ} \mathrm{C}$ (Wu et al, 2019). Surprisingly, at $25^{\circ} \mathrm{C}$, we found that the atfs-1(et17) mutant had a small decrease in resistance to PA14, whereas atfs-1(gk3094) mutants exhibited a small increase in resistance to PA14 compared with wild-type worms (Fig $4 \mathrm{H}$ ). At $20^{\circ} \mathrm{C}$, both atfs-1(gk3094) and atfs-1(et17) mutants had a small increase in resistance to PA14 compared with wild-type worms (Fig 4I). 
A

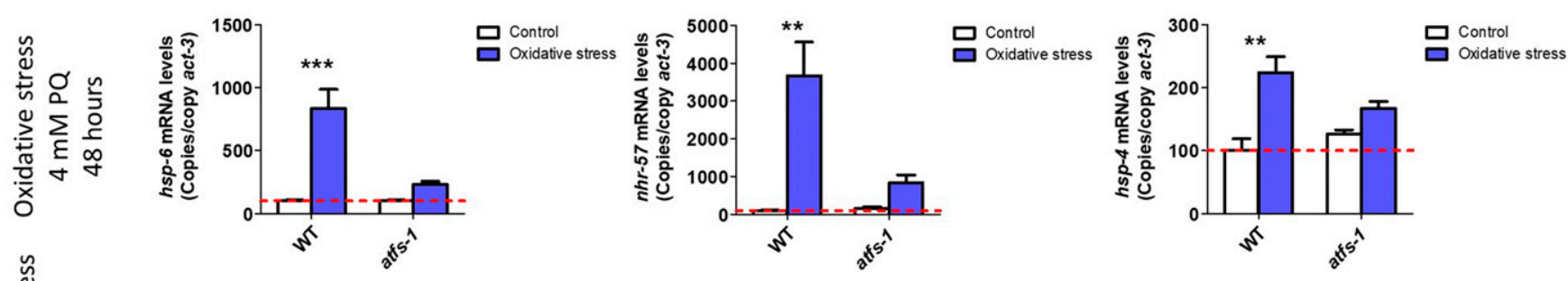

B
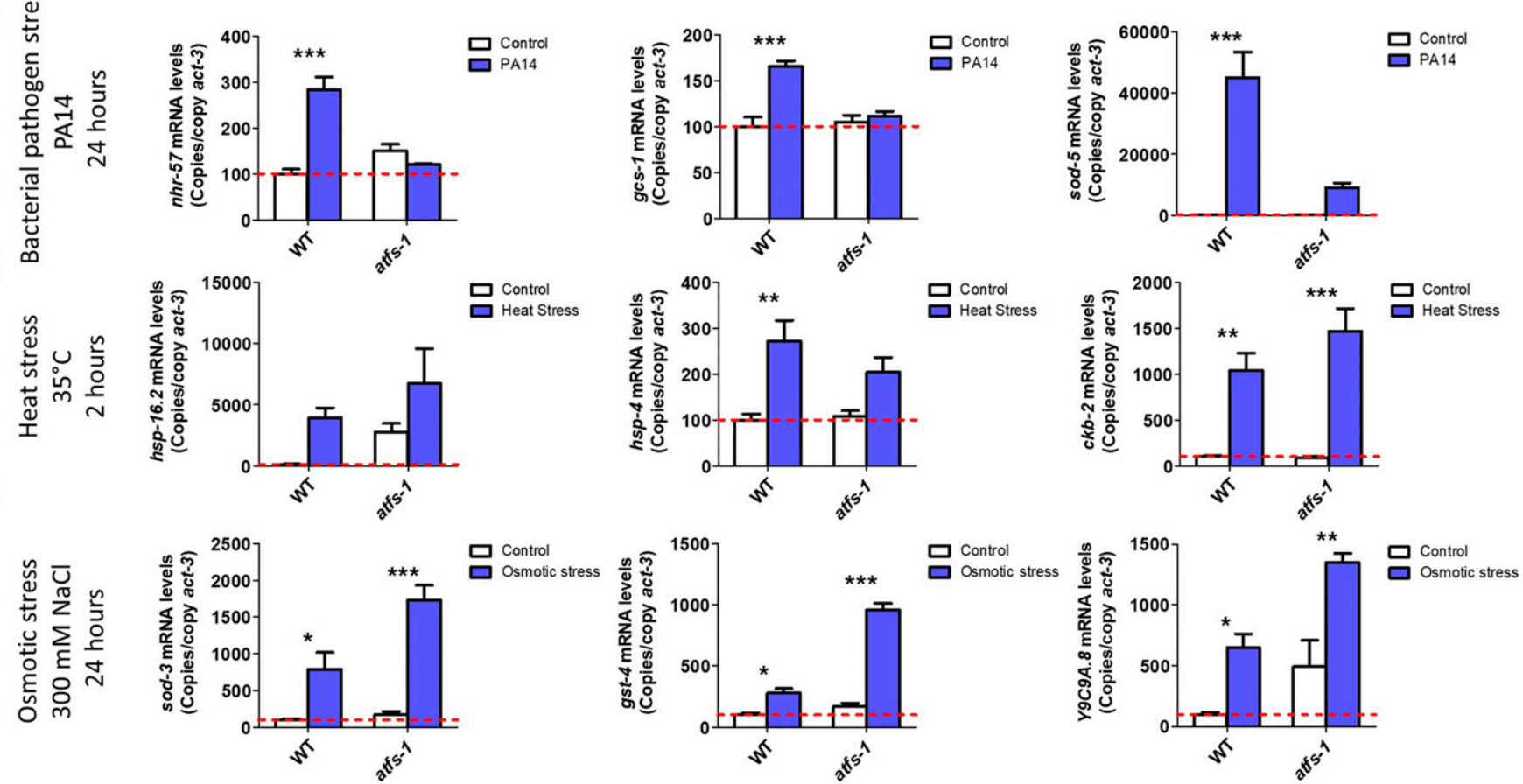

$\mathbf{E}$
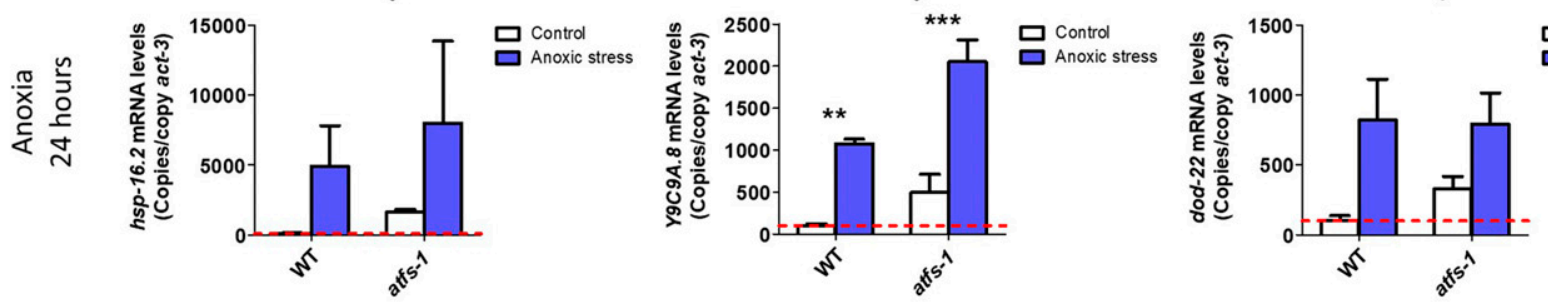

$\mathbf{F}$
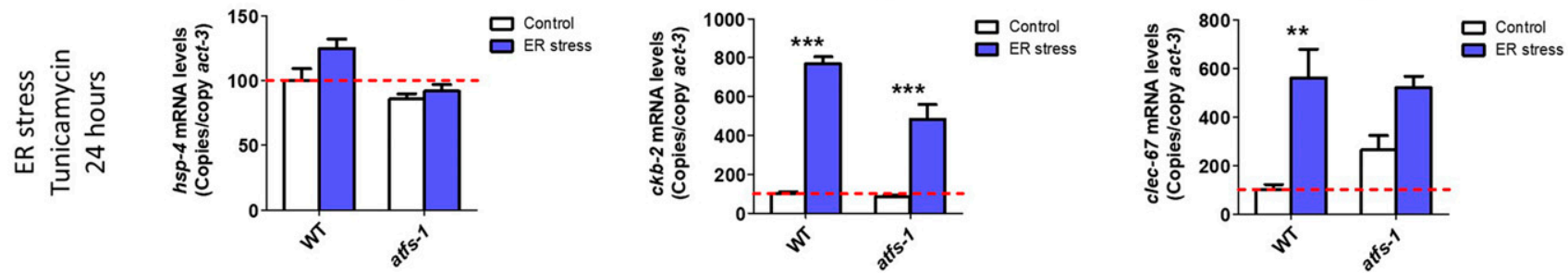

Figure 3. ATFS-1 is required for up-regulation of stress response genes after exposure to oxidative stress or bacterial pathogen stress.

To determine the role of ATFS-1 in responding to different types of stress, we compared the up-regulation of stress response genes in wild-type and atfs-1(gk3094) lossof-function deletion mutants after exposure to different stressors. (A) Exposure to oxidative stress ( $4 \mathrm{mM}$ paraquat, $48 \mathrm{~h}$ ) caused a significant up-regulation of $h \mathrm{sp}-6$, $\mathrm{nhr}$ 57 , and trx-2 in wild-type worms that was prevented by the disruption of atfs-1. (B) Exposure to bacterial pathogen stress (PA14, $24 \mathrm{~h}$ ) resulted in an up-regulation of nhr-57, gcs-1 and sod-5 in wild-type worms that was prevented by the atfs-1 deletion. (C) Exposure to heat stress $\left(35^{\circ} \mathrm{C}, 2 \mathrm{~h}\right)$ caused increased expression of $c k b-2$ and a trend towards increased expression of $h s p-16.2$ and $h s p-4$ in both wild-type and atfs-1 worms. (D) Exposure to osmotic stress (300 mM, $24 \mathrm{~h}$ ) caused an up-regulation of sod-3, gst-4, and Y9C9A.8 in wild-type worms and to a greater magnitude in atfs-1 mutants. (E) Anoxia (24 h) resulted in the up-regulation of hsp-16.2, Y9C9A.8, and dod-22 in both wild-type and atfs-1 worms. (F) Exposing worms to ER stress $(5 \mu \mathrm{g} / \mathrm{ml}$ tunicamycin, $24 \mathrm{~h}$ ) increased the expression of $c k b-2$ and trended towards increasing the expression of clec-67 in both wild-type and atfs-1 worms. Data information: Error bars indicate SEM. ${ }^{*} P<0.05,{ }^{* *} P<0.01,{ }^{* \star \star} P<0.001$. Statistical analysis was performed using a two-way ANOVA with a Bonferroni post hoc test. The number of replicates and statistical analysis can be found in Table S6. 

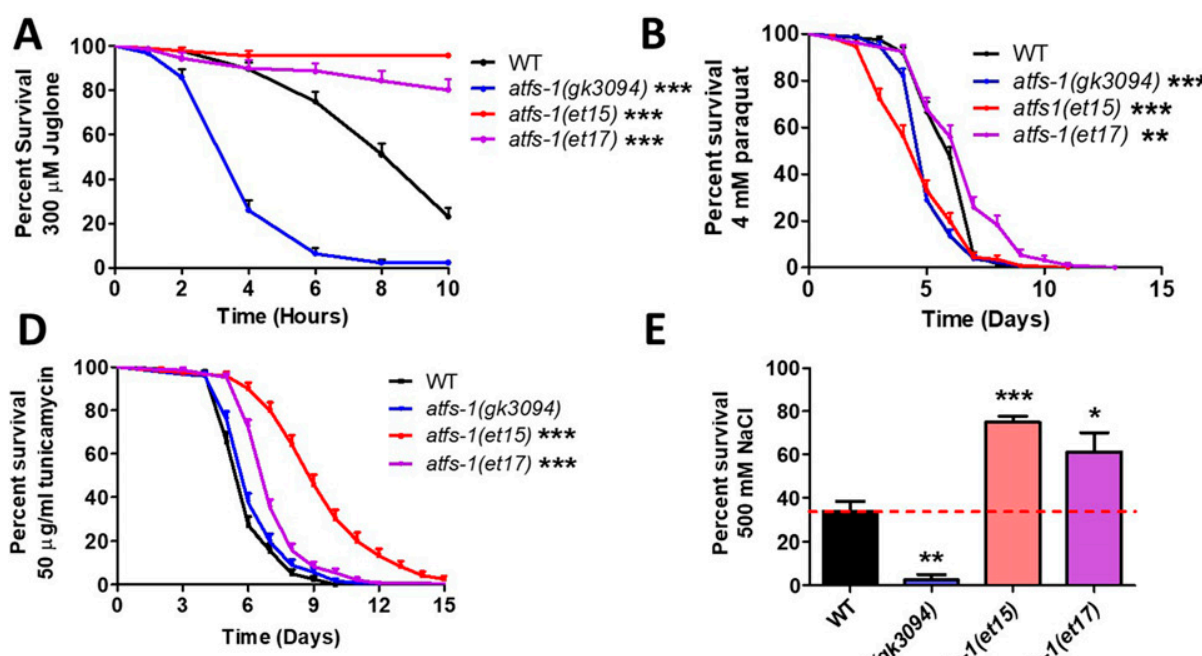

E
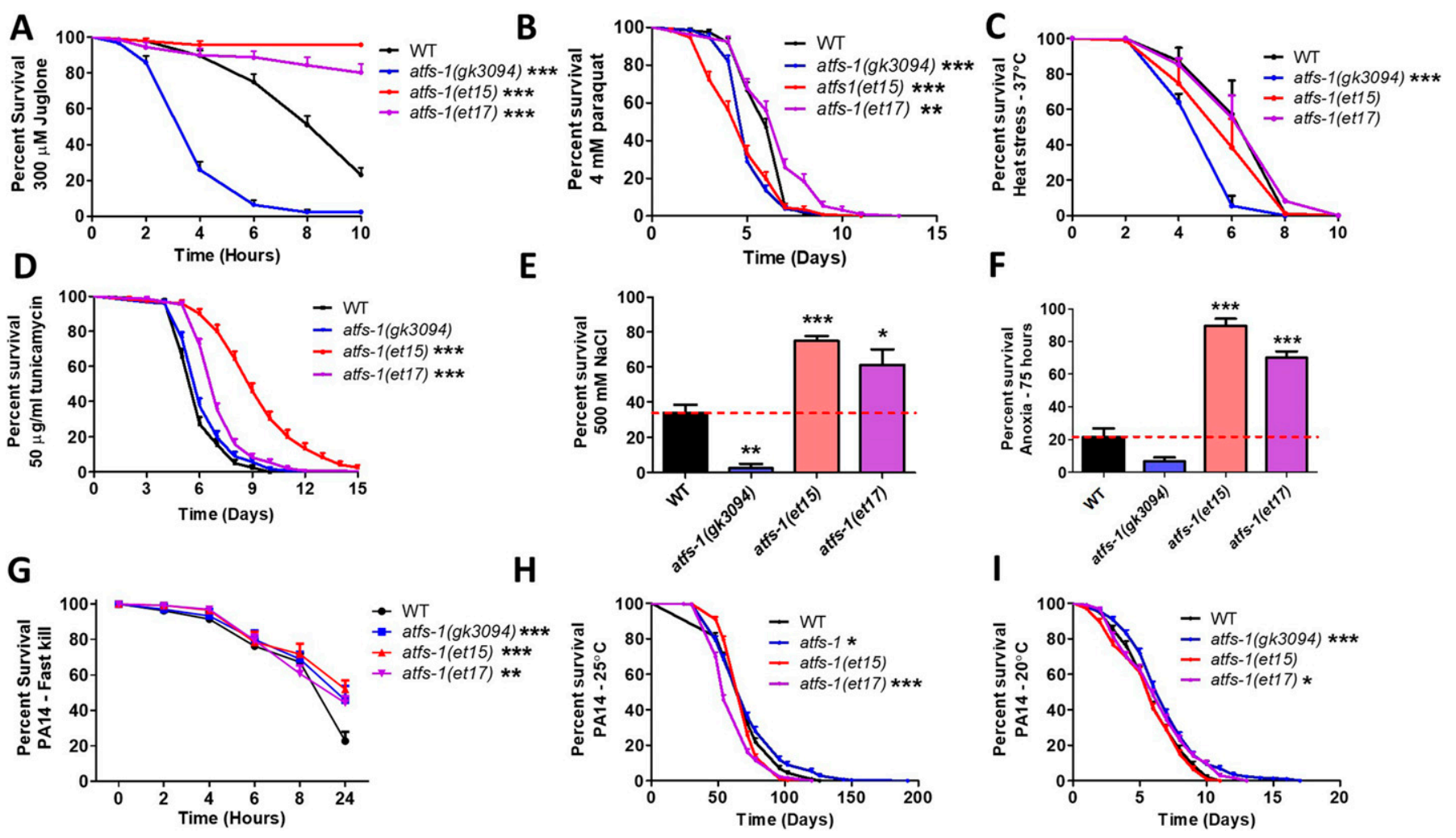

Figure 4. Constitutive activation of ATFS-1 increases resistance to multiple external stressors.

To determine the role of ATFS- 1 in resistance to stress, the stress resistance of an atfs-1 loss-of-function mutants (atfs-1( $\mathrm{k} k 3094)$ ) and two constitutively active atfs-1 gain-of-function mutants (atfs-1(et15), atfs-1(et17)) was compared with wild-type worms. (A) Activation of ATFS-1 enhanced resistance to acute oxidative stress (300 $\mu \mathrm{M}$ juglone), whereas disruption of atfs-1 markedly decreased resistance to acute oxidative stress. (B) Disruption of atfs-1 decreased resistance to chronic oxidative stress (4 mM paraquat). atfs-1(et17) mutants showed increased resistance to chronic oxidative stress, whereas atfs-1(et15) mutants had decreased resistance. (C) Resistance to heat stress $\left(37^{\circ} \mathrm{C}\right)$ was not enhanced by activation of ATFS-1, whereas disruption of atfs- 1 decreased heat stress resistance. (D) Constitutive activation of ATFS- 1 increased resistance to ER stress ( $50 \mu \mathrm{M}$ tunicamycin), whereas disruption of atfs- 1 had no effect. (E) Activation of ATFS- 1 increased resistance to osmotic stress ( 500 mM NaCl), whereas disruption of atfs-1 decreased osmotic stress resistance. (F) Constitutively active atfs-1 mutants show increased resistance to anoxia (75 h), whereas atfs-1 deletion mutants exhibit a trend towards decreased anoxia resistance. (G) Activation of ATFS-1 increased resistance to Pseudomonas aeruginosa toxin in a fast kill assay. A slow kill assay in which worms die from internal accumulation of $P$. aeruginosa was performed according to two established protocols. ( $\mathbf{H})$ At $25^{\circ} \mathrm{C}$, atfs-1(et17) mutants showed a small decrease in resistance to bacterial pathogens (PA14), wheras atfs-1(gk3094) mutants showed a small increase in resistance. (I) At 20 ${ }^{\circ} \mathrm{C}$, both atfs-1(et17) and atfs-1(gk3094) mutants exhibited a small increase in resistance to bacterial pathogens. Data for WT and atfs-1(gk3094) in panel (I) are from Campos et al (2021) as these strains were used as controls for two separate experiments that were performed at the same time. Data information: Error bars indicate $S E M$. ${ }^{*} P<0.05,{ }^{* *} P<0.01$, ${ }^{* * *} P<0.001$. Statistical analysis for panels $(\mathrm{A}, \mathrm{B}, \mathrm{D}, \mathrm{H}, \mathrm{I})$ were performed using the log-rank test. Statistical analysis for panels $\mathrm{C}$ and $\mathrm{G}$ were performed using a two-way ANOVA with Bonferroni post hoc test. Statistical analysis for panels (E, F) was performed using a one-way ANOVA with Bonferroni post hoc test. The number of replicates, $\mathrm{N}$, and statistical analysis can be found in Table S6.

All together, these data indicate that activation of ATFS-1 can protect against oxidative stress, ER stress, osmotic stress, anoxia, and bacterial pathogens but not heat stress. They also show that ATFS-1 is required for resistance to oxidative stress, heat stress, osmotic stress, and anoxia in wild-type worms.

\section{Long-lived genetic mutants up-regulate ATFS-1 target genes}

We previously showed that ATFS-1 target genes are up-regulated in three long-lived mitochondrial mutants: clk-1, isp-1, and nuo-6 (Lakowski \& Hekimi, 1996; Feng et al, 2001; Yang \& Hekimi, 2010b; Wu et al, 2018). To determine if ATFS-1 target genes are specifically up-regulated in long-lived mitochondrial mutants, or if they are also up-regulated in other long-lived mutants, we compared genes upregulated by ATFS-1 activation with gene expression in six additional long-lived mutants, which act through other longevity-promoting pathways. These long-lived mutants included sod-2 mutants, which act through increasing mitochondrial ROS (Van Raamsdonk \& Hekimi, 2009); daf-2 mutants, which have reduced insulin/IGF1 signaling (Kenyon et al, 1993); glp-1 mutants, which have germ line ablation (Hsin \& Kenyon, 1999); ife-2 mutants, which have reduced translation (Hansen et al, 2007); osm-5 mutants, which have reduced chemosensation (Apfeld \& Kenyon, 1999); and eat2 mutants, which have dietary restriction (Lakowski \& Hekimi, 1998).

After identifying differentially expressed genes in each of these long-lived mutants, we compared the differentially expressed genes with genes up-regulated by ATFS-1 activation. We defined ATFS-1-upregulated genes in two ways: (1) genes that are up-regulated by spg-7 RNAi in an ATFS-1-dependent manner (Nargund et al, 2012) and (2) genes that are up-regulated in a constitutively active atfs-1 mutant (et15; [Wu et al, 2018]). 
The majority of the long-lived mutants examined had a significant enrichment of ATFS-1 target genes. Genes up-regulated by spg-7 RNAi in an ATFS-1-dependent manner were significantly enriched in clk-1 mutants (6.7-fold enrichment), isp-1 mutants (6.0fold enrichment), sod-2 mutants (5.5-fold enrichment), nuo-6 mutants (4.1-fold enrichment), daf-2 mutants (2.6-fold enrichment), glp-1 mutants (2.0-fold enrichment), and ife-2 mutants (1.5-fold enrichment) (Fig 5). We did not find a significant enrichment of spg7 RNAi-induced ATFS-1 targets in osm-5 and eat-2 worms (Fig 5). Similarly, genes up-regulated in the constitutively active atfs-1(et15) mutant were significantly enriched in isp-1 mutants (3.5-fold enrichment), sod-2 mutants (3.4-fold enrichment), clk-1 mutants (3.3-fold enrichment), nuo-6 mutants (2.5-fold enrichment), daf-2 mutants (2.4-fold enrichment), glp-1 mutants (1.8-fold enrichment), ife-2 mutants (1.8-fold enrichment), and eat-2 mutants (1.5-fold enrichment) (Fig S9). We did not observe a significant enrichment of ATFS-1 target genes in osm-5 mutants (Fig S9).

Overall, these results indicate that ATFS- 1 target genes are upregulated in multiple long-lived mutants, including mutants in which mitochondrial function is not directly disrupted. Interestingly, in six of the seven strains exhibiting a significant enrichment of ATFS-1-modulated genes (all except ife-2, where the role of ROS has not been tested), there is an increase in ROS that contributes to their longevity as treatment with antioxidants decreases their lifespan (Van Raamsdonk \& Hekimi, 2009; Yang \& Hekimi, 2010a; Zarse et al, 2012; Wei \& Kenyon, 2016). This observation is consistent with the idea that ROS/oxidative stress is sufficient to activate the mitoUPR. As we have previously shown that exposure to a mild heat stress $\left(35^{\circ} \mathrm{C}, 2 \mathrm{~h}\right)$ or osmotic stress $(300 \mathrm{mM}, 24 \mathrm{~h})$ can extend lifespan but does not increase expression of the ATFS-1 target gene hsp-6 (Dues et al, 2016), it appears that only specific genes or interventions that extend longevity result in the up-regulation of ATFS-1 target genes.

\section{Constitutively active atfs-1 mutants have decreased lifespan despite enhanced resistance to stress}

Having shown that ATFS-1 target genes are activated in multiple long-lived mutants, we sought to determine if ATFS-1 activation is sufficient to increase lifespan and whether the presence of ATFS-1 is required for normal longevity in wild-type worms. Despite having increased resistance to multiple stressors, both constitutively active atfs-1 mutants (et15 and et17) have decreased lifespan compared with wild-type worms (Fig 6A and B), which is consistent with a previous study finding shortened lifespan in atfs-1(et17) and atfs1(et18) worms (Bennett et al, 2014). Despite having decreased resistance to multiple stressors, atfs-1 deletion mutants ( $\mathrm{gk3094}$ ) had a lifespan comparable with wild-type worms (Fig 6C), as we previously observed (Wu et al, 2018). Combined, this indicates that constitutive activation of ATFS-1 does not increase lifespan in a wild-type background, despite having an important role in stress resistance.

\section{Discussion}

Mitochondria are vital for organismal health as they perform multiple crucial functions within the cell including energy generation, metabolic

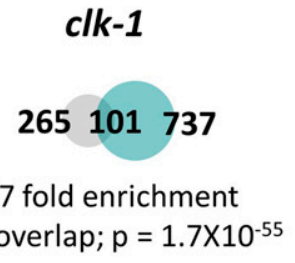

nuo-6

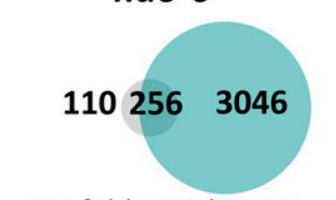

4.3 fold enrichment $70 \%$ overlap; $p=2.8 \times 10^{-119}$

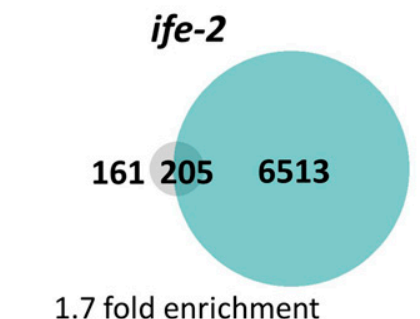

$56 \%$ overlap; $p=2.7 \times 10^{-20}$ isp-1

2011651344

6.1 fold enrichment $45 \%$ overlap; $p=2.6 \times 10^{-89}$

\section{daf-2}

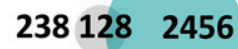

2.8 fold enrichment

$35 \%$ overlap; $p=7.5 \times 10^{-29}$

osm-5

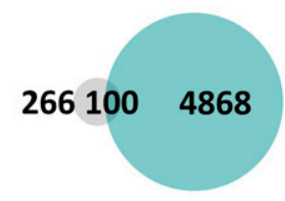

No enrichment

$27 \%$ overlap; $p=N S$ sod-2

32937331

5.6 fold enrichment $10 \%$ overlap; $p=1.7 \times 10^{-17}$

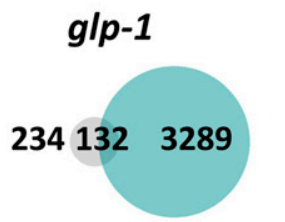

2.2 fold enrichment $36 \%$ overlap; $p=1.6 \times 10^{-19}$

\section{eat-2}

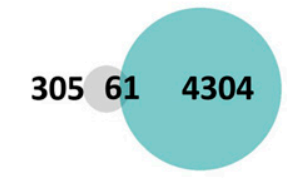

No enrichment $17 \%$ overlap; $p=0.015$
Figure 5. Multiple long-lived mutants from different pathways of lifespan extension show up-regulation of ATFS-1-dependent genes.

To determine the extent to which long-lived genetic mutants from different pathways of lifespan extension show differential expression of ATFS- 1 target genes, we compared genes that are up-regulated in nine different long-lived mutants to a published list of spg-7 RNAi-up-regulated, ATFS-1-dependent target genes (Nargund et al, 2012). clk-1, isp-1, nuo-6, sod-2, daf-2, glp-1, and ife-2 worms all show a highly significant degree of overlap with genes up-regulated by spg-7 RNAi in an ATFS-1-dependent manner. The grey circles represent the 366 genes that are upregulated by spg-7 RNAi in an ATFS-1-dependent manner. Turquoise circles are genes that are significantly up-regulated in the indicated long-lived mutant based upon our RNA sequencing data. The number of unique and overlapping genes is indicated. Percent overlap is calculated as the number of genes in common between the two gene sets divided by the total number of genes that are up-regulated by spg-7 RNAi in an ATFS-1-dependent manner. Enrichment is calculated as the number of overlapping genes observed divided by the number of overlapping genes predicted if genes were chosen randomly. P-values indicate the significance of the difference between the observed number of overlapping genes between the two gene sets, and the expected number of overlapping genes if the genes were picked at random. 


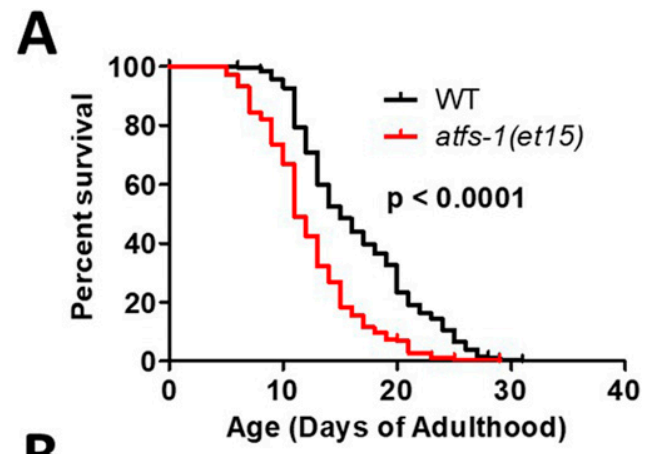

B

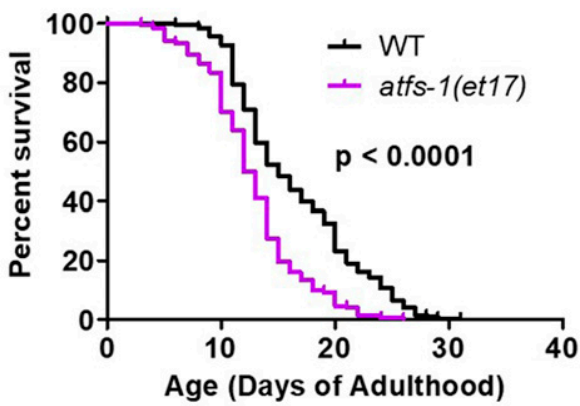

C

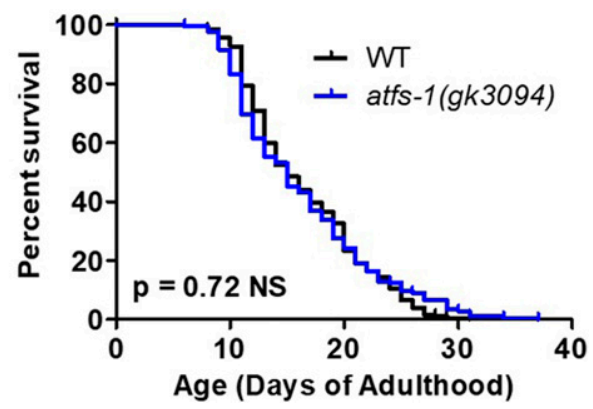

Figure 6. Activation of ATFS-1 does not increase lifespan.

To determine the effect of ATFS-1 on aging, we quantified the lifespan of an atfs-1 deletion mutant and two constitutively active atfs-1 mutants. (A, B) Both constitutively active atfs-1 mutants, et15 and et17, have a significantly decreased lifespan compared with wild-type worms. (C) Disruption of atfs-1 does not affect lifespan compared with wild-type worms. atfs-1(gk3094) is a loss of function mutant resulting from a deletion. atfs-1(et15) and atfs-1(et17) are constitutively active gain-of-function mutants. Data information: Statistical analysis was performed using the log-rank test. Statistical analysis, number of replicates, $\mathrm{N}$, and raw lifespan data are available in Table S6.

reactions, and intracellular signaling. Therefore, maintenance of mitochondrial function during times of acute stress and throughout normal aging is important for cell and organismal survival. The mitoUPR is a conserved pathway that facilitates restoration of mitochondrial homeostasis after internal or external stressors. In this work, we demonstrate a crucial role for the mitoUPR transcription factor ATFS-1 in the genetic response to external stressors, which ultimately promotes survival of the organism.

Throughout these studies, we utilized two different constitutively active atfs-1 mutants-et15 and et17. These two mutants contain point mutations in the MTS and differ only by one two amino acids (et15: G6E, et17: R4H) (Rauthan et al, 2013). Although atfs-1(et15) and atfs-1(et17) mutants generally behave similarly, they do exhibit differences, most notably in resistance to chronic oxidative stress and resistance to bacterial pathogens in the slow kill assay. These differences may result from atfs-1(et15) mutants having more extensive changes in gene expression than atfs-1(et17) mutants $(6,227$ differentially expressed genes versus 958 differentially expressed genes) (Wu et al, 2018). The et15 mutation may be more disruptive to the MTS than et17, thereby resulting in increased nuclear localization and more widespread changes in gene expression.

\section{ATFS-1 is not required for normal longevity}

A number of studies have directly or indirectly examined the role of the mitoUPR and ATFS-1 in longevity. In these studies, activation of the mitoUPR was typically measured using a mitoUPR reporter strain expressing GFP under the promoter of $h s p-6$, which is a target gene of ATFS-1 and the mitoUPR.

A relationship between the mitoUPR and longevity was first supported by the observation that disruption of the mitochondrial electron transport chain due to RNAi knockdown of the cytochrome $c$ oxidase-1 (cco-1) gene resulted in both activation of the mitoUPR (Yoneda et al, 2004; Durieux et al, 2011) and increased lifespan (Dillin et al, 2002). Since then, other lifespan-extending mutations have also been shown to activate the mitoUPR, including three long-lived mitochondrial mutants, clk-1, isp-1, and nuo-6 (Wu et al, 2018).

To explore this relationship in a more comprehensive manner, Runkel et al (2014) compiled a list of genes that activate the mitoUPR and examined their effect on lifespan. Of the 99 genes reported to activate the mitoUPR, 58 genes result in increased lifespan, although only 7 result in decreased lifespan (Runkel et al, 2014). Bennet et al (2014) performed an RNAi screen to identify RNAi clones that increase expression of a mitoUPR reporter strain (hsp-6p:: GFP) and quantified the effect of a selection of the mitoUPR-inducing clones on lifespan (Bennett et al, 2014). Of the 19 examined RNAi clones, 10 RNAi clones increased lifespan, while 6 decreased lifespan (Bennett et al, 2014). Using a similar approach to screen for compounds that activate a mitoUPR reporter strain ( $h s p-6 p:: G F P)$, metolazone was identified as a compound that activates the mitoUPR and extends lifespan in an ATFS-1-dependent manner (Ito et al, 2021). Combined, these results indicate that there are multiple genes or interventions which activate the mitoUPR and extend longevity, but there are also instances where these phenotypes are uncoupled.

Multiple experiments including the present study have examined the effect of the mitoUPR on lifespan directly by either increasing or decreasing the expression of components of the mitoUPR. RNAi knockdown of atfs-1 expression does not decrease wild-type lifespan (Bennett et al, 2014; Tian et al, 2016; Wu et al, 2018) nor do deletions in the atfs-1 gene decrease wild-type lifespan (Fig 6; [Bennett et al, 2014; Wu et al, 2018]). Thus, despite mitoUPR activation being correlated with longevity, ATFS-1 is not required for normal lifespan in a wild-type animal.

\section{ATFS-1 mediates lifespan extension in long-lived mutants}

Although ATFS-1 is dispensable for wild-type lifespan, it is required for lifespan extension of multiple long-lived mutants. Longevity can be extended by disrupting mito-nuclear protein balance through 
knocking down the expression of mitochondrial ribosomal protein S5 ( $m r s p-5)$, which also increases the expression of the mitoUPR target gene $h s p-6$. The magnitude of the lifespan extension caused by mrsp-5 RNAi is decreased by knocking down the key mitoUPR component gene haf-1 or ubl-5 (Houtkooper et al, 2013). In the long-lived mitochondrial mutant nuo-6, disruption of atfs-1 completely reverts the long lifespan to wild-type length, and treatment with atfs-1 RNAi has similar effects (Wu et al, 2018). In the mitochondrial mutant isp-1, knocking down a key initiator of mitoUPR, ubl-5, decreases their long lifespan but has no effect on the lifespan of wild-type worms (Durieux et al, 2011). In contrast, it has been reported that knockdown of atfs-1 using RNAi does not decrease isp-1 lifespan (Bennett et al, 2014). However, it is possible that in the latter study, the magnitude of knockdown may not have been sufficient to have effects on lifespan as lifelong exposure to atfs-1 RNAi prevents larval development of isp-1 worms (Baker et al, 2012; Wu et al, 2018). Similarly, differing results have been obtained for the requirement of the mitoUPR in the extended lifespan resulting from cco-1 knockdown. Although it has been reported that mutation of atfs-1 does not decrease the lifespan of worms treated

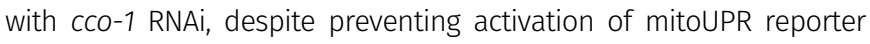
(Bennett et al, 2014), a subsequent study found that atfs-1 RNAi decreases the extent of lifespan extension resulting from cco-1 RNAi (Tian et $\mathrm{al}$, 2016). Although differing results have been observed in some cases, overall, these studies suggest that ATFS- 1 and the mitoUPR have a role in mediating the lifespan extension in a subset of long-lived mutants.

Despite the fact that long-lived mutants with chronic activation of the mitoUPR depend on ATFS-1 for their long lifespan, our current results using the constitutively active atfs-1(et15) and atfs-1(et17) mutants, as well as previous results using constitutively active atfs1 mutants (et17 and et18), show that constitutive activation of ATFS-1 in wild-type worms results in decreased lifespan (Fig 6) (Bennett et al, 2014). This may be partially due to activation of ATFS-1, increasing the proportion of damaged mtDNA when heteroplasmy exists (Lin et al, 2016). Consistent with this finding, overexpression of the mitoUPR target gene hsp-60 also leads to a small decrease in lifespan (Jeong et al, 2017). In contrast, overexpression of a different mitoUPR target gene, hsp-6, is sufficient to increase lifespan (Yokoyama et al, 2002). It has also been shown that a hypomorphic reduction-of-function mutation allele of hsp-6 (mg583) also increases lifespan, whereas hsp-6 null mutations are thought to be lethal (Mao et al, 2019). Combined, these results indicate that chronic activation of the mitoUPR is mildly detrimental for wild-type lifespan, but that modulation of specific target genes can be beneficial.

It is important to note that the lifespan assays completed in this study and previous studies were completed under laboratory conditions, which are believed to be relatively unstressed. It is possible that constitutive activation of ATFS-1 may increase lifespan in an uncontrolled environment where worms encounter external stressors, as observed with our various stress assays. The magnitude of ATFS-1 activation may impact its effect on stress resistance and lifespan. Perhaps, a milder activation of ATFS-1 will be more beneficial with respect to lifespan, which could be determined through dose-response experiments involving RNAimediated knockdown of atfs-1 in the constitutively active atfs-1 mutants.

\section{ATFS-1 is necessary for stress resistance in wild-type animals}

Although ATFS-1 is not required for longevity in wild-type animals, it plays a significant role in protecting animals against exogenous stressors. Disrupting atfs-1 function decreases organismal resistance to oxidative stress, heat stress, osmotic stress, and anoxia (Fig 4). Additionally, we previously determined that inhibiting atfs- 1 in long-lived nuo-6 worms completely suppressed the increased resistance to oxidative stress, osmotic stress, and heat stress typically observed in that mutant (Wu et al, 2018) and that disruption of atfs-1 in Parkinson's disease mutants $p d r-1$ and pink-1 decreased their resistance to oxidative stress, osmotic stress, heat stress, and anoxia (Cooper et al, 2017). Combined, these results demonstrate that ATFS-1 is required for resistance to multiple exogenous stressors.

Even though ATFS-1 is required for the up-regulation of stress response genes in response to bacterial pathogens (Fig 3), disruption of atfs-1 (gk3094 mutation) did not decrease bacterial pathogen resistance. Similarly, another atfs-1 deletion mutation (tm4919) was found not to affect survival during exposure to $P$. aeruginosa (Pellegrino et al, 2014). In contrast, Jeong et al (2017) did observe decreased bacterial pathogen survival in atfs1(gk3094) mutants (Jeong et al, 2017). Knocking down atfs-1 through RNAi also inconsistently decreased survival on P. aeruginosa (e.g., Fig 3A versus Fig 3H in Pellegrino et al [2014]). It is unclear why disruption of atfs-1 has a variable effect on bacterial pathogen resistance but may result from subtle differences in the way the assay is conducted.

Consistent with our finding that atfs-1 deletion does not decrease resistance to bacterial pathogens in wild-type worms, we have shown that baseline expression of innate immunity genes in wild-type animals is also not affected by disruption of atfs-1 (Campos et al, 2021). In contrast, disrupting genes involved in the p38-mediated innate immune signaling pathway does decrease resistance to bacterial pathogens and does decrease the expression of innate immunity genes in a wild-type background (Campos et al, 2021). Combined, this indicates that baseline levels of innate immunity gene expression and bacterial pathogen resistance are dependent on the p38-mediated innate immune signaling pathway and are not dependent on ATFS-1. In contrast, the expression of innate immunity genes can be enhanced by activation of ATFS-1, either in nuo- 6 mutants (Campos et al, 2021) or constitutively active atfs-1 mutants (Fig 4G).

Decreasing the expression of a downstream ATFS-1 target gene, hsp-60, by RNAi caused a robust decrease in organismal survival on $P$. aeruginosa (Jeong et al, 2017). As we have previously found that disrupting atfs-1 induces up-regulation of other protective cellular pathways (Wu et al, 2018), and others have observed a similar phenomenon when a mitoUPR downstream target, $h s p-6$, is disrupted (Kim et al, 2016), it is possible that the up-regulation of other stress pathways may compensate for the inhibition of the mitoUPR in atfs-1 deletion mutants, ultimately yielding wild-type or increased levels of resistance to bacterial pathogens and hiding the normal role of the mitoUPR in resistance to bacterial pathogens. 


\section{Activation of ATFS-1 enhances resistance to exogenous stressors}

In this work, we show that constitutive activation of ATFS-1 (atfs1(et15) and atfs-1(et17) mutants) is sufficient to increase resistance to multiple different exogenous stressors, including oxidative stress, ER stress, osmotic stress, anoxia, and bacterial pathogens. Previous studies have shown that activating the mitoUPR, either through spg-7 RNAi or through a constitutively active atfs-1(et15) mutant, decreased risk of death after anoxia-reperfusion (Pena et al, 2016) and that constitutively active atfs-1(et18) mutants have increased resistance to $P$. aeruginosa (Pellegrino et al, 2014). Overexpression of the mitoUPR target gene hsp-60 also increases resistance to $P$. aeruginosa (Jeong et al, 2017). These results support a clear role for ATFS-1 in surviving external stressors.

Although ATFS-1 activation protects against multiple external stressors, not all of these stressors activate ATFS-1. Previously, we exposed a mitoUPR reporter strain (hsp-6p::GFP) to heat stress, cold stress, osmotic stress, anoxia, oxidative stress, starvation, ER stress, and bacterial pathogens, and only oxidative stress increased mitoUPR activity (Dues et al, 2016). As the constitutively active atfs-1 mutants (et15 and et17) exhibit activation of the mitoUPR under unstressed conditions (e.g., up-regulation of hsp-6 in Fig $1 \mathrm{~A}$; up-regulation of many other stress pathway target genes Fig 2; increased fluorescence of $h s p-6$ and $h s p-60$ reporter strains in Rauthan et al [2013]), it is likely that the activation of the mitoUPR and downstream stress response pathways under unstressed conditions is primarily responsible for the increased resistance to stress that we observe in the constitutively active atfs-1 mutants.

\section{ATFS-1 up-regulates target genes of multiple stress response pathways}

In exploring the mechanism by which ATFS-1 and the mitoUPR modulate stress resistance, we found that activation of ATFS-1, through mild impairment of mitochondrial function (nuo-6) or through constitutive activation of ATFS-1 (atfs-1(et15)), causes upregulation of genes involved in multiple stress response pathways, including the ER-UPR pathway, the Cyto-UPR pathway, the DAF16-mediated stress response pathway, the SKN-1-mediated oxidative stress response pathway, the HIF-mediated hypoxia response pathway, the p38-mediated innate immune response pathway, and antioxidant genes (Fig 2). These findings are consistent with those of earlier work demonstrating a role for ATFS-1 in up-regulating innate immunity genes. Pellegrino et al (2014) reported a 16\% (59/365 genes) overlap between genes up-regulated by activation of the mitoUPR through treatment with spg-7 RNAi and genes up-regulated by exposure a bacterial pathogen (Pellegrino et al, 2014). A connection between the mitoUPR and the innate immunity pathway was also suggested by the finding that overexpression of a mitoUPR downstream target, $h s p-60$, increases expression of three innate immunity genes: T24B8.5/sysm-1, C17H12.8, and K08D8.5 (Jeong et al, 2017). Our results clearly indicate that the role of ATFS- 1 in stress response pathways is not limited to the innate immunity but extends to multiple stress response pathways, thereby providing a mechanistic basis for the effect of ATFS-1 on resistance to stress.
Although our results do not definitively distinguish between direct or indirect regulation of genes and other stress response pathways by ATFS-1, analysis of previous CHiP-seq experiments demonstrates that ATFS-1 can bind to the same genes as other stress-responsive transcription factors including HSF-1, DAF-16, HIF-1, SKN-1, and ATF-7. The ability of ATFS- 1 to bind to these genes suggests that ATFS-1 may be able to directly regulate a subset of target genes of other stress response pathways.

\section{Conclusions}

The mitoUPR is required for animals to survive exposure to exogenous stressors, and activation of this pathway is sufficient to enhance resistance to stress (Table S5). In addition to upregulating genes involved in restoring mitochondrial homeostasis, the mitoUPR increases stress resistance by up-regulating the target genes of multiple stress response pathways. Although increased stress resistance has been associated with long lifespan, and multiple long-lived mutants exhibit activation of the mitoUPR, constitutive activation of ATFS-1 shortens lifespan while increasing resistance to stress, indicating that the role of ATFS-1 in stress resistance can be experimentally dissociated from its role in longevity. Overall, this work highlights the importance of the mitoUPR in not only protecting organisms from internal stressors but also improving organismal survival upon exposure to external stressors

\section{Materials and Methods}

\section{Strains}

C. elegans strains were obtained from the Caenorhabditis Genetics Center (CGC): N2 (wild-type), nuo-6(qm200), atfs-1(gk3094), nuo6(qm200);atfs-1(gk3094), atfs-1(et15), atfs-1(et17), ife-2 (ok306), clk1(qm30), sod-2(ok1030), eat-2(ad1116), osm-5(p813), isp-1(qm150), daf-2(e1370), and glp-1(e2141). Strains were maintained at $20^{\circ} \mathrm{C}$ on nematode growth medium (NGM) plates seeded with OP50 Escherichia coli. atfs-1(et15) and atfs-1(et17) were outcrossed 10 times (Rauthan et al, 2013), and atfs-1(gk3094) were outcrossed six times. Young adult worms are picked on day 1 of adulthood before egg laying begins. The worms were not synchronized but picked visually as close to the L4-adult transition as possible.

\section{Gene expression in response to stress}

\section{Stress treatment}

Young adult worms were subject to different stress before mRNA was collected. For heat stress, worms were incubated at $35^{\circ} \mathrm{C}$ for $2 \mathrm{~h}$ and $20^{\circ} \mathrm{C}$ for $4 \mathrm{~h}$. For oxidative stress, worms were transferred to plates containing $4 \mathrm{mM}$ paraquat and $1000 \mu \mathrm{M}$ FUdR for $48 \mathrm{~h}$. FUdR was used for these samples because (1) with the 2-d duration of this stress, worms can produce progeny which would complicate the collection of the experimental worms; and (2) $4 \mathrm{mM}$ paraquat often results in internal hatching of progeny when FUdR is absent, which might have affected the results. Because FUdR has the potential to 
alter gene expression, the control worms for the 48-h $4 \mathrm{~mm}$ paraquat stress were also treated with $100 \mu \mathrm{M}$ FUdR. For ER stress, worms were transferred to plates containing $5 \mu \mathrm{g} / \mathrm{ml}$ tunicamycin for $24 \mathrm{~h}$. For osmotic stress, worms were transferred to plates containing $300 \mathrm{mM}$ $\mathrm{NaCl}$ and left for $24 \mathrm{~h}$. For bacterial pathogen stress, worms were transferred to plates seeded with P. aeruginosa strain PA14 and left for $4 \mathrm{~h}$. For anoxic stress, worms were put in BD Bio-Bag Type A Environmental Chambers (Becton, Dickinson and Company) for $24 \mathrm{~h}$ and left to recover for $4 \mathrm{~h}$. For unstressed control conditions, worms were collected at the young adult stage for heat stress and bacterial pathogens; $24 \mathrm{~h}$ after the young adult stage for osmotic stress, ER stress and anoxia; and $48 \mathrm{~h}$ after the young adult stage for oxidative stress.

\section{RNA isolation}

RNA was harvested, as described previously (Schaar et al, 2015). The plates of worms were washed three times using M9 buffer to remove bacteria and resuspended in TRIzol reagent. Worms were frozen in a dry ice/methanol bath and then thawed three times and left at room temperature for $15 \mathrm{~min}$. Chloroform was added to the tubes, and the mixture was left to sit at room temperature for $3 \mathrm{~min}$. The tubes were then centrifuged at $12,000 \mathrm{~g}$ for $15 \mathrm{~min}$ at $4^{\circ} \mathrm{C}$. The upper phase containing the RNA was transferred to a new tube, mixed with isopropanol, and allowed to sit at room temperature for 10 $\min$. The tubes were centrifuged at $12,000 \mathrm{~g}$ for $10 \mathrm{~min}$ at $4^{\circ} \mathrm{C}$. The RNA pellet was washed with $75 \%$ ethanol and resuspended in RNAse-free water.

\section{Quantitative RT-PCR}

mRNA was converted to cDNA using a High-Capacity cDNA Reverse Transcription kit (Life Technologies/Invitrogen), as described previously (Machiela et al, 2016). qPCR was performed using a PowerUp SYBR Green Master Mix kit (Applied Biosystems) in a Viia 7 RT-PCR machine from Applied Biosystems. All experiments were performed with at least three biological replicates collected from different days. mRNA levels were normalized to act-3 levels and then expressed as percentage of wild-type. Primer sequences are as follows:

gst-4 (CTGAAGCCAACGACTCCATT, GCGTAAGCTTCTTCCTCTGC), hsp-4 (CTCGTGGAATCAACCCTGAC, GACTATCGGCAGCGGTAGAG), hsp-6 (CGCTGGAGATAAGATCATCG, TTCACGAAGTCTCTGCATGG), hsp-16.2 (CCATCTGAGTCTTCTGAGATTGTT, CTTTCTTTGGCGCTTCAATC), sod-3 (TACTGCTCGCACTGCTTCAA, CATAGTCTGGGCGGACATTT), sod-5 (TTCCACAGGACGTTGTTTCC, ACCATGGAACGTCCGATAAC), nhr-57 (GACTCTGTGTGGAGTGATGGAGAG, GTGGCTCTTGGTGTCAATTTGGG), gCs-1 (CCACCAGATGCTCCAGAAAT, TGCATTTTCAAAGTCGGTC), trx-2 (GTTGATTTCCACGCAGAATG, TGGCGAGAAGAACACTTCCT), Y9C9A.8 (CGGGGATATAACTGATAGAATGG, CAAACTCTCCAGCTTCCAACA), T24B8.5 (TACACTGCTTCAGAGTCGTG, CGACAACCACTTCTAACATCTG), clec-67 (TTTGGCAGTCTACGCTCGTT, CTCCTGGTGTGTCCCATTTT), dod-22 (TCCAGGATACAGAATACGTACAAGA, GCCGTTGATAGTTTCGGTGT), ckb-2 (GCATTTATCCGAGACAGCGA, GCTTGCACGTCCAAATCAAC), act-3 (TGCGACATTGATATCCGTAAGG, GGTGGTTCCTCCGGAAAGAA).

\section{RNA sequencing and bioinformatics analysis}

RNA sequencing was performed previously (Dues et al, 2017; Senchuk et al, 2018), and raw data are available on the National
Center for Biotechnology Information (NCBI) Gene Expression Omnibus (GEO): GSE93724 (Senchuk et al, 2018), GSE110984 (Wu et al, 2018). Bioinformatics analysis for this study was used to determine differentially expressed genes and identify the degree and significance of overlaps between genes sets.

\section{Determining differentially expressed genes}

Samples were processed using an RNA-seq pipeline based on the bcbio-nextgen project (https:/ / bcbio-nextgen.readthedocs.org/ en/latest/). We examined raw reads for quality issues using FastQC (http://www.bioinformatics.babraham.ac.uk/projects/fastqc/) in order to ensure library generation, and sequencing data were suitable for further analysis. If necessary, we used cutadapt https:// cutadapt.readthedocs.io/en/stable/ to trim adapter sequences, contaminant sequences such as polyA tails, and low-quality sequences from reads. We aligned trimmed reads to the Ensembl build WBcel235 (release 90) of the C. elegans genome using STAR (Dobin et al, 2013). We assessed the quality of alignments by checking for evenness of coverage, ribosomal RNA content, genomic context of alignments (e.g., alignments in known transcripts and introns), complexity, and other quality checks. To quantify expression, we used Salmon (Patro et al, 2017) to find transcript-level abundance estimates and then collapsed down to the gene level using the R Bioconductor package tximport (Soneson et al, 2015). Principal components analysis and hierarchical clustering methods were used to validate clustering of samples from the same batches and across different mutants. We used the R Bioconductor package DESeq2 (Love et al, 2014) to find differential expression at the gene level. For each wild-type mutant comparison, we identified significant genes with an false discovery rate threshold of 0.01. Lastly, we included batch as a covariate in the linear model for datasets in which experiments were run across two batches.

\section{Venn diagrams}

Weighted Venn diagrams were produced by inputting gene lists into BioVenn (https://www.biovenn.nl/). Percentage overlap was determined by dividing the number of genes in common between the two gene sets by the gene list with the smaller gene list.

\section{Significance of overlap and enrichment}

The significance of overlap between two gene sets was determined by comparing the actual number of overlapping genes with the expected number of overlapping genes based on the sizes of the two gene sets (expected number $=$ number of genes in set $1 \times$ number of genes in set 2 /number of genes in genome detected). Enrichment was calculated as the observed number of overlapping genes/the expected number of overlapping genes if genes were chosen randomly.

\section{Resistance to stress}

For acute oxidative stress, young adult worms were transferred onto plates with $300 \mu \mathrm{M}$ juglone and survival was measured every 2 $\mathrm{h}$ for a total of $10 \mathrm{~h}$. For chronic oxidative stress, young adult worms were transferred onto plates with $4 \mathrm{mM}$ paraquat and $100 \mu \mathrm{M}$ FUdR and survival was measured daily until death. 
For heat stress, young adult worms were incubated in $37^{\circ} \mathrm{C}$ and survival was measured every $2 \mathrm{~h}$ for a total of $10 \mathrm{~h}$. For osmotic stress, young adult worms were transferred to plates containing 450 or $500 \mathrm{mM} \mathrm{NaCl}$ and survival was measured after $48 \mathrm{~h}$. For anoxic stress, plates with young adult worms were put into BD Bio-Bag Type A Environmental Chambers for $75 \mathrm{~h}$ and survival was measured after a $24-h$ recovery period.

Resistance to ER stress was tested by transferring young adult worms to agar plates containing either $50 \mu \mathrm{g} / \mathrm{ml}$ tunicamycin (654380; EMD Millipore) in 0.5\% DMSO (472301; Sigma-Aldrich) or $0.5 \%$ DMSO only at $20^{\circ} \mathrm{C}$. Survival was measured every day until death.

Two different bacterial pathogenesis assays involving P. aeruginosa strain PA14 were performed. In the slow kill assay, worms are thought to die from intestinal colonization of the pathogenic bacteria, whereas in the fast kill assay, worms are thought to die from a toxin secreted from the bacteria (Kirienko et al, 2014). The slow kill assay was performed, as described previously (Pellegrino et al, 2014; Wu et al, 2019). In the first protocol (Pellegrino et al, 2014), PA14 cultures were grown overnight and seeded to center of a 35$\mathrm{mm}$ NGM agar plate. The plates were left to dry overnight and then incubated in $37^{\circ} \mathrm{C}$ for $24 \mathrm{~h}$. The plates were left to adjust to room temperature before $\sim 40 \mathrm{~L} 4$ worms were transferred onto the plates. The assay was conducted $25^{\circ} \mathrm{C}$, and the plates were checked twice a day until death. In the second protocol (Wu et al, 2019), overnight PA14 culture were seeded to the center of a 35-mm NGM agar plate containing $20 \mathrm{mg} / \mathrm{l}$ FUdR. The plates were incubated at $37^{\circ} \mathrm{C}$ overnight and then at room temperature overnight before $\sim 40 \mathrm{~d}$, three adults were transferred onto these plates. The assay was conducted $20^{\circ} \mathrm{C}$, and the plates were checked daily until death. The fast kill pathogenesis assay was performed, as described previously (Kirienko et al, 2014). PA14 cultures were grown overnight and seeded to peptone-glucose-sorbitol agar plates. Seeded plates were left to dry for $20 \mathrm{~min}$ at room temperature before incubation at $37^{\circ} \mathrm{C}$ for $24 \mathrm{~h}$ and then at $23^{\circ} \mathrm{C}$ for another $24 \mathrm{~h}$. Approximately $30 \mathrm{~L} 4$ worms were transferred onto the plates and were scored as dead or alive at $2,4,6,8$, and $24 \mathrm{~h}$. Fast kill plates were kept at $23^{\circ} \mathrm{C}$ in between scoring time points.

\section{Lifespan}

All lifespan assays were performed at $20^{\circ} \mathrm{C}$. Lifespan assays included FUdR to limit the development of progeny and the occurrence of internal hatching. Based on our previous studies, a low concentration of FUdR (25 mM) was used to minimize potential effects of FUdR on lifespan (Van Raamsdonk \& Hekimi, 2011). Animals were excluded from the experiment if they crawled off the plate or died of internal hatching of progeny or expulsion of internal organs.

\section{Statistical analysis}

All of our statistical analyses are provided in Table 56 including the number of replicates, worms per replicate, statistical test utilized, and all $P$-values. To ensure unbiased results, all experiments were conducted with the experimenter blinded to the genotype of the worms. For all assays, a minimum of three biological replicates of randomly selected worms from independent populations of worms on different days were used. For analysis of lifespan, oxidative stress, and bacterial pathogen stress, a log-rank test was used. For analysis of heat stress, repeated measures ANOVA was used. For analysis of osmotic stress and anoxic stress, a one-way ANOVA with Dunnett's multiple comparisons test was used. For quantitative PCR results, we used a two-way ANOVA with the Bonferroni post hoc test. For all bar graphs, error bars indicate the standard error of the mean and bars indicate the mean.

\section{Data Availability}

RNA-seq data have been deposited on GEO: GSE93724, GSE110984. All other data and strains generated in the current study are included with the article or available from the corresponding author on request.

\section{Supplementary Information}

Supplementary Information is available at https://doi.org/10.26508/lsa. 202101182.

\section{Acknowledgements}

Some strains were provided by the CGC, which is funded by the National Institutes of Health ( $\mathrm{NIH}$ ) Office of Research Infrastructure Programs (P30 OD010440). We would also like to acknowledge the C. elegans knockout consortium and the National Bioresource Project of Japan for providing strains used in this research. This work was supported by the Canadian Institutes of Health Research (CIHR; http://www.cihr-irsc.gc.ca/; JM Van Raamsdonk), the Natural Sciences and Engineering Research Council of Canada (NSERC; https://www.nserc-crsng.gc.ca/index_eng.asp; JM Van Raamsdonk), and the National Institute of General Medical Sciences (NIGMS; https://www.nigms.nih.gov/; JM Van Raamsdonk) by grant number R01 GM121756. JM Van Raamsdonk is the recipient of a Senior Research Scholar career award from the Fonds de Recherche du Québec Santé (FRQS) and Parkinson Quebec. A Traa received scholarships from the NSERC and FRQS SK Soo received a scholarship from the FRQS. PD Rudich received a fellowship award from the FRQS. The funders had no role in study design, data collection and analysis, decision to publish, or preparation of the manuscript.

\section{Author Contributions}

SK Soo: formal analysis, investigation, visualization, methodology, and writing-original draft, review, and editing.

A Traa: formal analysis, investigation, visualization, methodology, and writing-review and editing.

PD Rudich: formal analysis, investigation, visualization, methodology, and writing-review and editing.

M Mistry: formal analysis, investigation, visualization, and methodology.

JM Van Raamsdonk: conceptualization, formal analysis, supervision, funding acquisition, investigation, visualization, methodology, project administration, and writing-original draft, review, and editing. 


\section{Conflict of Interest Statement}

The authors declare that they have no conflict of interest.

\section{References}

Apfeld J, Kenyon C (1999) Regulation of lifespan by sensory perception in Caenorhabditis elegans. Nature 402: 804-809. doi:10.1038/45544

Baker BM, Nargund AM, Sun T, Haynes CM (2012) Protective coupling of mitochondrial function and protein synthesis via the elF2 $\alpha$ kinase GCN-2. PLoS Genet 8: e1002760. doi:10.1371/journal.pgen.1002760

Bennett CF, Kaeberlein M (2014) The mitochondrial unfolded protein response and increased longevity: Cause, consequence, or correlation? Exp Gerontol 56: 142-146. doi:10.1016/j.exger.2014.02.002

Bennett CF, Vander Wende H, Simko M, Klum S, Barfield S, Choi H, Pineda VV, Kaeberlein M (2014) Activation of the mitochondrial unfolded protein response does not predict longevity in Caenorhabditis elegans. Nat Commun 5: 3483. doi:10.1038/ncomms4483

Berendzen KM, Durieux J, Shao LW, Tian Y, Kim HE, Wolff S, Liu Y, Dillin A (2016) Neuroendocrine coordination of mitochondrial stress signaling and proteostasis. Cell 166: 1553-1563.e10. doi:10.1016/j.cell.2016.08.042

Bishop T, Lau KW, Epstein AC, Kim SK, Jiang M, O'Rourke D, Pugh CW, Gleadle JM, Taylor MS, Hodgkin J, et al (2004) Genetic analysis of pathways regulated by the von hippel-lindau tumor suppressor in Caenorhabditis elegans. PLoS Biol 2: e289. doi:10.1371/ journal.pbio.0020289

Cacho-Valadez B, Muñoz-Lobato F, Pedrajas JR, Cabello J, Fierro-González JC, Navas P, Swoboda P, Link CD, Miranda-Vizuete A (2012) The characterization of the Caenorhabditis elegans mitochondrial thioredoxin system uncovers an unexpected protective role of thioredoxin reductase 2 in $\beta$-amyloid peptide toxicity. Antioxid Redox Signal 16: 1384-1400. doi:10.1089/ars.2011.4265

Campos JC, Wu Z, Rudich PD, Soo SK, Mistry M, Ferreira JCB, Blackwell TK, Van Raamsdonk JM (2021) Mild impairment of mitochondrial function increases longevity and pathogen resistance through atfs-1-driven activation of p38-regulated innate immunity. EMBO Rep. doi:10.15252/ embr.202152964

Cooper JF, Machiela E, Dues DJ, Spielbauer KK, Senchuk MM, Van Raamsdonk JM (2017) Activation of the mitochondrial unfolded protein response promotes longevity and dopamine neuron survival in Parkinson's disease models. Sci Rep 7: 16441. doi:10.1038/s41598-017-16637-2

Dillin A, Hsu AL, Arantes-Oliveira N, Lehrer-Graiwer J, Hsin H, Fraser AG, Kamath RS, Ahringer J, Kenyon C (2002) Rates of behavior and aging specified by mitochondrial function during development. Science 298 : 2398-2401. doi:10.1126/science.1077780

Dobin A, Davis CA, Schlesinger F, Drenkow J, Zaleski C, Jha S, Batut P, Chaisson M, Gingeras TR (2013) Star: Ultrafast universal rna-seq aligner. Bioinformatics 29: 15-21. doi:10.1093/bioinformatics/bts635

Dues DJ, Andrews EK, Schaar CE, Bergsma AL, Senchuk MM, Van Raamsdonk JM (2016) Aging causes decreased resistance to multiple stresses and a failure to activate specific stress response pathways. Aging (Albany NY) 8: 777-795. doi:10.18632/aging.100939

Dues DJ, Schaar CE, Johnson BK, Bowman MJ, Winn ME, Senchuk MM, Van Raamsdonk JM (2017) Uncoupling of oxidative stress resistance and lifespan in long-lived isp-1 mitochondrial mutants in Caenorhabditis elegans. Free Radic Biol Med 108: 362-373. doi:10.1016/ j.freeradbiomed.2017.04.004

Durieux J, Wolff S, Dillin A (2011) The cell-non-autonomous nature of electron transport chain-mediated longevity. Cell 144: 79-91. doi:10.1016/ j.cell.2010.12.016
Ewald CY, Landis JN, Porter Abate J, Murphy CT, Blackwell TK (2015) Dauerindependent insulin/igf-1-signalling implicates collagen remodelling in longevity. Nature 519: 97-101. doi:10.1038/nature14021

Feng J, Bussière F, Hekimi S (2001) Mitochondrial electron transport is a key determinant of life span in Caenorhabditis elegans. Dev Cell 1: 633-644. doi:10.1016/s1534-5807(01)00071-5

Fiorese CJ, Schulz AM, Lin Y-F, Rosin N, Pellegrino MW, Haynes CM (2016) The transcription factor atf5 mediates a mammalian mitochondrial upr. Curr Biol 26: 2037-2043. doi:10.1016/j.cub.2016.06.002

Fletcher M, Tillman EJ, Butty VL, Levine SS, Kim DH (2019) Global transcriptional regulation of innate immunity by atf-7 in C. elegans. PLoS Genet 15: e1007830. doi:10.1371/journal.pgen.1007830

Hansen M, Taubert S, Crawford D, Libina N, Lee SJ, Kenyon C (2007) Lifespan extension by conditions that inhibit translation in Caenorhabditis elegans. Aging cell 6: 95-110. doi:10.1111/j.1474-9726.2006.00267.x

Honda Y, Honda S (1999) The daf-2 gene network for longevity regulates oxidative stress resistance and mn-superoxide dismutase gene expression in Caenorhabditis elegans. FASEB J 13: 1385-1393. doi:10.1096/fasebj.13.11.1385

Houtkooper RH, Mouchiroud L, Ryu D, Moullan N, Katsyuba E, Knott G, Williams RW, Auwerx J (2013) Mitonuclear protein imbalance as a conserved longevity mechanism. Nature 497: 451-457. doi:10.1038/ nature12188

Hsin H, Kenyon C (1999) Signals from the reproductive system regulate the lifespan of C. elegans. Nature 399: 362-366. doi:10.1038/20694

Ito A, Zhao Q, Tanaka Y, Yasui M, Katayama R, Sun S, Tanimoto Y, Nishikawa Y, Kage-Nakadai E (2021) Metolazone upregulates mitochondrial chaperones and extends lifespan in Caenorhabditis elegans. Biogerontology 22: 119-131. doi:10.1007/s10522-020-09907-6

Jeong DE, Lee D, Hwang SY, Lee Y, Lee JE, Seo M, Hwang W, Seo K, Hwang AB, Artan M, et al (2017) Mitochondrial chaperone hsp-60 regulates antibacterial immunity via p38 map kinase signaling. EMBO / 36: 1046-1065. doi:10.15252/embj.201694781

Jovaisaite V, Mouchiroud L, Auwerx J (2014) The mitochondrial unfolded protein response, a conserved stress response pathway with implications in health and disease. J Exp Biol 217: 137-143. doi:10.1242/ jeb.090738

Kahn NW, Rea SL, Moyle S, Kell A, Johnson TE (2008) Proteasomal dysfunction activates the transcription factor skn-1 and produces a selective oxidative-stress response in Caenorhabditis elegans. Biochem J 409: 205-213. doi:10.1042/BJ20070521

Kenyon C, Chang J, Gensch E, Rudner A, Tabtiang R (1993) A C. elegans mutant that lives twice as long as wild type. Nature 366: 461-464. doi:10.1038/ $366461 a 0$

Kim HE, Grant AR, Simic MS, Kohnz RA, Nomura DK, Durieux J, Riera CE, Sanchez M, Kapernick E, Wolff S, et al (2016) Lipid biosynthesis coordinates a mitochondrial-to-cytosolic stress response. Cell 166: 1539-1552.e16. doi:10.1016/j.cell.2016.08.027

Kirienko NV, Cezairliyan BO, Ausubel FM, Powell JR (2014) Pseudomonas aeruginosa pa14 pathogenesis in Caenorhabditis elegans. Methods Mol Biol 1149: 653-669. doi:10.1007/978-1-4939-0473-0_50

Kovács D, Sigmond T, Hotzi B, Bohár B, Fazekas D, Deák V, Vellai T, Barna J (2019) Hsf1base: A comprehensive database of hsf1 (heat shock factor 1) target genes. Int J Mol Sci 20: 5815. doi:10.3390/ijms20225815

Kudron MM, Victorsen A, Gevirtzman L, Hillier LW, Fisher WW, Vafeados D, Kirkey M, Hammonds AS, Gersch J, Ammouri H, et al (2018) The modern resource: Genome-wide binding profiles for hundreds of drosophila and Caenorhabditis elegans transcription factors. Genetics 208: 937-949. doi:10.1534/genetics.117.300657

Kumar N, Jain V, Singh A, Jagtap U, Verma S, Mukhopadhyay A (2015) Genomewide endogenous DAF-16/FOXO recruitment dynamics during 
lowered insulin signalling in C. elegans. Oncotarget 6: 41418-41433. doi:10.18632/oncotarget.6282

Lakowski B, Hekimi S (1996) Determination of life-span in Caenorhabditis elegans by four clock genes. Science 272: 1010-1013. doi:10.1126/ science.272.5264.1010

Lakowski B, Hekimi S (1998) The genetics of caloric restriction in Caenorhabditis elegans. Proc Natl Acad Sci U S A 95: 13091-13096. doi:10.1073/pnas.95.22.13091

Li J, Chauve L, Phelps G, Brielmann RM, Morimoto RI (2016) E2f coregulates an essential hsf developmental program that is distinct from the heatshock response. Genes Dev 30: 2062-2075. doi:10.1101/gad.283317.116

Lin Y-F, Schulz AM, Pellegrino MW, Lu Y, Shaham S, Haynes CM (2016) Maintenance and propagation of a deleterious mitochondrial genome by the mitochondrial unfolded protein response. Nature 533: 416-419. doi:10.1038/nature17989

Link CD, Cypser JR, Johnson CJ, Johnson TE (1999) Direct observation of stress response in Caenorhabditis elegans using a reporter transgene. Cell Stress Chaperones 4: 235-242. doi:10.1379/1466-1268(1999)004<0235: doosri>2.3.co;2

Love MI, Huber W, Anders S (2014) Moderated estimation of fold change and dispersion for rna-seq data with deseq2. Genome Biol 15: 550. doi:10.1186/s13059-014-0550-8

Machiela E, Dues DJ, Senchuk MM, Van Raamsdonk JM (2016) Oxidative stress is increased in C. elegans models of huntington's disease but does not contribute to polyglutamine toxicity phenotypes. Neurobiol Dis 96: 1-11. doi:10.1016/j.nbd.2016.08.008

Mao K, Ji F, Breen P, Sewell A, Han M, Sadreyev R, Ruvkun G (2019) Mitochondrial dysfunction in C. elegans activates mitochondrial relocalization and nuclear hormone receptor-dependent detoxification genes. Cell Metab 29: 1182-1191.e4. doi:10.1016/ j.cmet.2019.01.022

Merkwirth C, Jovaisaite V, Durieux J, Matilainen O, Jordan SD, Quiros PM, Steffen KK, Williams EG, Mouchiroud L, Tronnes SU, et al (2016) Two conserved histone demethylases regulate mitochondrial stressinduced longevity. Cell 165: 1209-1223. doi:10.1016/j.cell.2016.04.012

Nargund AM, Fiorese CJ, Pellegrino MW, Deng P, Haynes CM (2015) Mitochondrial and nuclear accumulation of the transcription factor ATFS-1 promotes OXPHOS recovery during the UPRmt. Mol cell 58: 123-133. doi:10.1016/j.molcel.2015.02.008

Nargund AM, Pellegrino MW, Fiorese CJ, Baker BM, Haynes CM (2012) Mitochondrial import efficiency of atfs-1 regulates mitochondrial upr activation. Science 337: 587-590. doi:10.1126/science.1223560

Niu W, Lu ZJ, Zhong M, Sarov M, Murray JI, Brdlik CM, Janette J, Chen C, Alves P, Preston E, et al (2011) Diverse transcription factor binding features revealed by genome-wide chip-seq in C. elegans. Genome Res 21: 245-254. doi:10.1101/gr.114587.110

Patro R, Duggal G, Love MI, Irizarry RA, Kingsford C (2017) Salmon provides fast and bias-aware quantification of transcript expression. Nat Methods 14: 417-419. doi:10.1038/nmeth.4197

Pellegrino MW, Nargund AM, Kirienko NV, Gillis R, Fiorese CJ, Haynes CM (2014) Mitochondrial upr-regulated innate immunity provides resistance to pathogen infection. Nature 516: 414-417. doi:10.1038/nature13818

Peña S, Sherman T, Brookes PS, Nehrke K (2016) The mitochondrial unfolded protein response protects against anoxia in Caenorhabditis elegans. PLoS One 11: e0159989. doi:10.1371/journal.pone.0159989

Rauthan M, Ranji P, Aguilera Pradenas N, Pitot C, Pilon M (2013) The mitochondrial unfolded protein response activator atfs-1 protects cells from inhibition of the mevalonate pathway. Proc Natl Acad Sci U S A 110: 5981-5986. doi:10.1073/pnas.1218778110

Runkel ED, Baumeister R, Schulze E (2014) Mitochondrial stress: Balancing friend and foe. Exp Gerontol 56: 194-201. doi:10.1016/ j.exger.2014.02.013
Schaar CE, Dues DJ, Spielbauer KK, Machiela E, Cooper JF, Senchuk M, Hekimi S, Van Raamsdonk JM (2015) Mitochondrial and cytoplasmic ros have opposing effects on lifespan. PLoS Genet 11: e1004972. doi:10.1371/ journal.pgen.1004972

Senchuk MM, Dues DJ, Schaar CE, Johnson BK, Madaj ZB, Bowman MJ, Winn ME, Van Raamsdonk JM (2018) Activation of daf-16/foxo by reactive oxygen species contributes to longevity in long-lived mitochondrial mutants in Caenorhabditis elegans. PLoS Genet 14: e1007268. doi:10.1371/ journal.pgen.1007268

Shen C, Nettleton D, Jiang M, Kim SK, Powell-Coffman JA (2005a) Roles of the hif-1 hypoxia-inducible factor during hypoxia response in Caenorhabditis elegans. J Biol Chem 280: 20580-20588. doi:10.1074/ jbc.M501894200

Shen X, Ellis RE, Sakaki K, Kaufman RJ (2005b) Genetic interactions due to constitutive and inducible gene regulation mediated by the unfolded protein response in C. elegans. PLoS Genet 1: e37. doi:10.1371/ journal.pgen.0010037

Shpilka T, Haynes CM (2018) The mitochondrial upr: Mechanisms, physiological functions and implications in ageing. Nat Rev Mol Cell Biol 19: 109-120. doi:10.1038/nrm.2017.110

Soneson C, Love MI, Robinson MD (2015) Differential analyses for rna-seq: Transcript-level estimates improve gene-level inferences. F1000Res 4 : 1521. doi:10.12688/f1000research.7563.2

Steinbaugh MJ, Narasimhan SD, Robida-Stubbs S, Moronetti Mazzeo LE, Dreyfuss JM, Hourihan JM, Raghavan P, Operaña TN, Esmaillie R, Blackwell TK (2015) Lipid-mediated regulation of skn-1/nrf in response to germ cell absence. Elife 4: e07836. doi:10.7554/eLife.07836

Sural S, Lu TC, Jung SA, Hsu AL (2019) Hsb-1 inhibition and hsf-1 overexpression trigger overlapping transcriptional changes to promote longevity in Caenorhabditis elegans. G3 (Bethesda) 9: 1679-1692. doi:10.1534/g3.119.400044

Tepper RG, Ashraf J, Kaletsky R, Kleemann G, Murphy CT, Bussemaker HJ (2013) Pqm-1 complements daf-16 as a key transcriptional regulator of daf2-mediated development and longevity. Cell 154: 676-690. doi:10.1016/ j.cell.2013.07.006

Tian Y, Garcia G, Bian Q, Steffen KK, Joe L, Wolff S, Meyer BJ, Dillin A (2016) Mitochondrial stress induces chromatin reorganization to promote longevity and upr(mt). Cell 165: 1197-1208. doi:10.1016/j.cell.2016.04.011

Urano F, Calfon M, Yoneda T, Yun C, Kiraly M, Clark SG, Ron D (2002) A survival pathway for Caenorhabditis elegans with a blocked unfolded protein response. J Cell Biol 158: 639-646. doi:10.1083/jcb.200203086

Van Raamsdonk JM, Hekimi S (2009) Deletion of the mitochondrial superoxide dismutase sod-2 extends lifespan in Caenorhabditis elegans. PLoS Genet 5: e1000361. doi:10.1371/journal.pgen.1000361

Van Raamsdonk JM, Hekimi S (2011) Fudr causes a twofold increase in the lifespan of the mitochondrial mutant gas-1. Mech Ageing Dev 132: 519-521. doi:10.1016/j.mad.2011.08.006

Webb AE, Kundaje A, Brunet A (2016) Characterization of the direct targets of foxo transcription factors throughout evolution. Aging cell 15 : 673-685. doi:10.1111/acel.12479

Wei Y, Kenyon C (2016) Roles for ros and hydrogen sulfide in the longevity response to germline loss in Caenorhabditis elegans. Proc Natl Acad Sci U S A 113: E2832-E2841. doi:10.1073/pnas.1524727113

Wu Z, Isik M, Moroz N, Steinbaugh MJ, Zhang P, Blackwell TK (2019) Dietary restriction extends lifespan through metabolic regulation of innate immunity. Cell Metab 29: 1192-1205.e8. doi:10.1016/j.cmet.2019.02.013

Wu Z, Senchuk MM, Dues DJ, Johnson BK, Cooper JF, Lew L, Machiela E, Schaar CE, Dejonge H, Blackwell TK, et al (2018) Mitochondrial unfolded protein response transcription factor atfs-1 promotes longevity in a long-lived mitochondrial mutant through activation of stress response pathways. BMC Biol 16: 147. doi:10.1186/s12915-018-0615-3 
Yang W, Hekimi S (2010a) A mitochondrial superoxide signal triggers increased longevity in Caenorhabditis elegans. PLoS Biol 8: e1000556. doi:10.1371/journal.pbio.1000556

Yang W, Hekimi S (2010b) Two modes of mitochondrial dysfunction lead independently to lifespan extension in Caenorhabditis elegans. Aging cell 9: 433-447. doi:10.1111/j.1474-9726.2010.00571.x

Yokoyama K, Fukumoto K, Murakami T, Harada S, Hosono R, Wadhwa R, Mitsui Y, Ohkuma S (2002) Extended longevity of Caenorhabditis elegans by knocking in extra copies of hsp70f, a homolog of mot-2 (mortalin)/ mthsp70/grp75. FEBS Lett 516: 53-57. doi:10.1016/s0014-5793(02)024705

Yoneda T, Benedetti C, Urano F, Clark SG, Harding HP, Ron D (2004) Compartment-specific perturbation of protein handling activates genes encoding mitochondrial chaperones. J Cell Sci 117: 4055-4066. doi:10.1242/jcs.01275
Zarse K, Schmeisser S, Groth M, Priebe S, Beuster G, Kuhlow D, Guthke R, Platzer M, Kahn CR, Ristow M (2012) Impaired insulin/igf1 signaling extends life span by promoting mitochondrial l-proline catabolism to induce a transient ros signal. Cell Metab 15: 451-465. doi:10.1016/ j.cmet.2012.02.013

Zhao Q, Wang J, Levichkin IV, Stasinopoulos S, Ryan MT, Hoogenraad NJ (2002) A mitochondrial specific stress response in mammalian cells. EMBO J 21: 4411-4419. doi:10.1093/emboj/cdf445

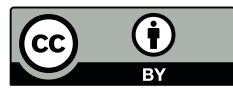

License: This article is available under a Creative Commons License (Attribution 4.0 International, as described at https://creativecommons.org/ licenses/by/4.0/). 